\title{
Unveiling non-Markovian spacetime signaling in open quantum systems with long-range tensor network dynamics
}

\author{
Thibaut Lacroix $\odot,{ }^{1,2,}{ }^{*}$ Angus Dunnett $\odot,{ }^{2}$ Dominic Gribben $\odot,{ }^{1}$ Brendon W. Lovett, ${ }^{1}$ and Alex Chin ${ }^{2}$ \\ ${ }^{1}$ SUPA, School of Physics and Astronomy, University of St Andrews, St Andrews KY16 9SS, United Kingdom \\ ${ }^{2}$ Institut des NanoSciences de Paris, CNRS, Sorbonne Université, 4 Place Jussieu, 75005 Paris, France
}

(Received 9 July 2021; revised 17 September 2021; accepted 5 October 2021; published 4 November 2021)

\begin{abstract}
Nanoscale devices, either biological or artificial, operate in a regime where the usual assumptions of a structureless Markovian bath do not hold. Being able to predict and study the dynamics of such systems is crucial and is usually done by tracing out the bath degrees of freedom, which implies losing information about the environment. To go beyond these approaches we use a numerically exact method relying on a matrix product state representation of the quantum state of a system and its environment to keep track of the bath explicitly. This method is applied to a specific example of interaction that depends on the spatial structure of a system made of two sites. The result is that we predict a non-Markovian dynamics where long-range couplings induce correlations into the environment. The environment dynamics can be naturally extracted from our method and shine a light on long-time feedback effects that are responsible for the observed non-Markovian recurrences in the eigenpopulations of the system.
\end{abstract}

DOI: 10.1103/PhysRevA.104.052204

\section{INTRODUCTION}

Real-life quantum systems are never truly isolated from the rest of the universe and are typically exposed to a macroscopic number of fluctuating degrees of freedom that constitute their often unobservable (and invariably uncontrollable) environments [1,2]. Weak interactions of a quantum system with spectrally broad and dynamically featureless environments lead to so-called Markovian dissipation in which energy relaxation and decoherence can be accurately described by a time-local Redfield or Lindblad master equation [1-3]. In these leaky systems, the perturbations of the environment caused by the system rapidly and irreversibly propagate away, essentially removing any trace, or memory, of prior interactions in the way the environment acts locally on the embedded system (see Fig. 1). Acting always in the instant and having no dependence on the shared history of the system-bath interactions, Markovian noise is thus very difficult to control, and most strategies to combat its unwanted effects simply aim at its total suppression.

However, in functional nanoscale materials the dividing line between the system and environmental excitations becomes less clear, and large and long-lasting correlations between them can build up over the duration of a process. In the presence of these nonequilibrium conditions, these correlations can, inter alia, lead to nonclassical work extraction, energy transport, and violation of detailed balance [4-6]. The investigation of how open system-environment correlations influence and might even help optimize energy harvesting, transport, and transduction processes in devices operating at

\footnotetext{
*tfml1@st-andrews.ac.uk
}

the few-quantum level is an important research line in the burgeoning field of quantum thermodynamics $[7,8]$.

Nowhere are these concepts of more relevance than in the protein-based nanomachines that nature has developed to perform the key optoelectronic tasks of photosynthesis. For example, the pigment-protein complexes (PPCs) that perform the electron transfers at the core of photosynthesis are composed of photoactive pigments in interaction with a highly structured environment made of a protein scaffold that tunes the electronic and vibrational properties of the molecular network. The structure of such a reaction center (RC) is shown in Fig. 2. The electron transport chain is shown in Fig. 2(b), beginning at the special pair of chlorophyll and terminating at the quinone acceptors (not shown). In higher plants, the hole left behind by electron transport is ultimately refilled by the splitting of water and evolution of oxygen [9]. This requires the RC being turned over four electrons in a concerted action, a remarkable feat of multicarrier photocatalysis.

Coordinating multiple charge dynamics in structures with poor dielectric screening and typical lateral sizes of only 5-6 $\mathrm{nm}$ requires exquisite spatiotemporal control of energy transfer and electron transport, including mechanisms of feedback to ensure the processes occur in the correct order without waste of excited-state energies. While the role of the structured environments that found PPCs has been widely discussed in terms of transport efficiency and the possible support of coherent electronic dynamics in light harvesting [10-13], the signaling and potential efficiency gains from spatiotemporal feedback and heralding feedforward processes in the environment have received rather scant attention. However, first-principles methods based on crystal structures do show that the large secondary protein elements that span the electron transport chain in the RC could communicate the initial and final sites of the electron transport and may act 
(a)

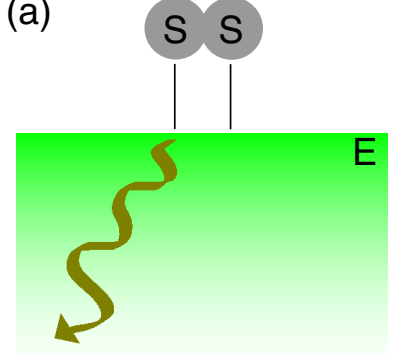

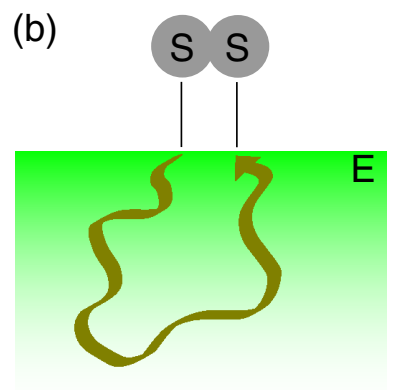

FIG. 1. Schematic representation of (a) a Markovian environment and (b) a non-Markovian environment. In a Markovian environment, excitations created through the interaction with the bath propagate away and do not influence the system. By contrast, in the non-Markovian case, these excitations can have a backaction at a later time on a different part of the system.

to prevent accumulation of further charges [14]. Elsewhere in biology, the idea of dynamical structural changes as a way to regulate processes is well established, especially in the field of allosteric regulation [15,16]. Considered as an open quantum system problem, the existence of strong spatiotemporal correlations necessitates a manifestly non-Markovian description of the dynamics, as the key physics is encoded in the retarded action at a distance that results from previous system-bath interactions, energy exchange, etc. In this article we develop a model that allows us to explore these effects in a fully quantum mechanical description which opens a route to establishing the phenomenology of non-Markovian dissipation in the regime where system dynamics, relaxation transitions, and environmental signaling occur on similar timescales. By first identifying and understanding the under-

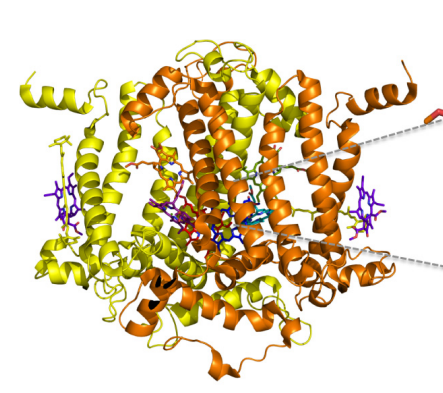

(a)

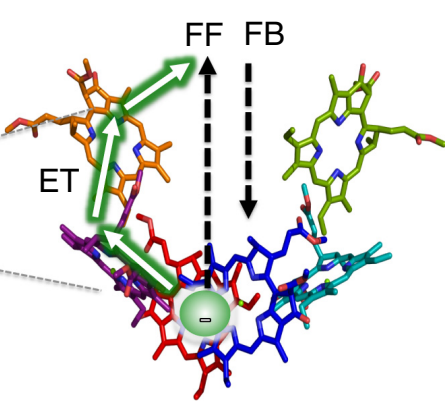

(b)
FIG. 2. Biological inspiration for our correlated bath model. (a) Protein structure of a nanoscale photosynthetic reaction center. Photoactive pigments are held rigidly by noncovalent protein interactions that also tune their electronic overlaps, interactions, and excited-state energies. The coordination of multiple cofactors by extended structures, such as quasi-one-dimensional $\alpha$ helices, allows vibrational fluctuations to act on different cofactors in a spatiotemporally correlated manner. (b) Structure of the cofactors active in charge separation through quantum electron transport (ET). The oxidation of water in photosynthesis requires four successful electron transports and this multifermion process is regulated through feedforward (FF) and feedback (FB) mechanisms induced by strong electron-hole interactions with the dissipative protein scaffold. lying microscopic physics behind these phenomena, we hope to build up a conceptual base that could be used to exploit these effects, including any explicitly nonclassical effects, in artificial nanoscale devices.

However, capturing non-Markovian dynamics has proven to be quite challenging because of the large amount of information that usually needs to be kept about the system's dynamics and the large number of (often continuous) modes in the environment which subjects such problems to the curse of dimensionality: The number of possible quantum states grows exponentially with the number of modes of the environment. Moreover, non-Markovian dynamics are also nonperturbative and their study thus requires the use of advanced numerical methods. There are two broad approaches to this problem, reduced density matrix methods and wave-function approaches. The former does not keep a microscopic description of the environment. The only information kept about the environment is its correlation function, or equivalently its spectral density. The evolution of the system's density matrix can then be described, for example, by an approximate weak-coupling master equation [1] or exactly using a process tensor [17] or a tensor network representation of the influence functional as in the time-evolving matrix product operator (TEMPO) method $[18,19]$. Indeed, a process tensor can be extracted from the TEMPO method [20] and this can lead to still more efficient calculations [21]. The latter distinct approach relies on a wave-function representation of the isolated joint system and keeps an explicit microscopic description of the environment, but often with an alternative description of its degrees of freedom. For example, the time-evolving density operator with orthonormal polynomials algorithm (TEDOPA) [22] maps the continuum of independent modes of the environment into a chain with nearest-neighbor couplings. Alternatively, the multilayer multiconfiguration time-dependent Hartree method [23] relies on a description of the environmental degrees of freedom with so-called time-dependent single-particle functions. Both the reduced density matrix and wave-function approaches have gained numerical efficiency by using tensor network Ansätze as their fundamental objects and exploiting efficient contractions and compression techniques.

In this paper we present an extension of the TEDOPA method to describe system-bath interactions that are long range even in the mapped chain topology. These long-range interactions come in our model from a spatial dependence of the phases of the coupling coefficients between sites of the system and the environment. We describe the properties of these new couplings and how they can be integrated in the usual matrix product operator (MPO) representation of the Hamiltonian in Sec. II C. Notably, with this method the new tensors of the MPO scale with the (small) dimension of the reduced system and are independent of the (large) dimension of the environment. The time evolution is then performed on a two-site system and its environment using a one-site time-dependent variational principle (1TDVP) [24] scheme with a matrix product state (MPS) representation of the wave function. Standard tensor network-based approaches, such as time-evolving block decimation (TEBD) [25], are formulated for local interactions and can treat long-range interactions only at the cost of an increased complexity (by increasing the number of steps needed to perform the time evolution, 


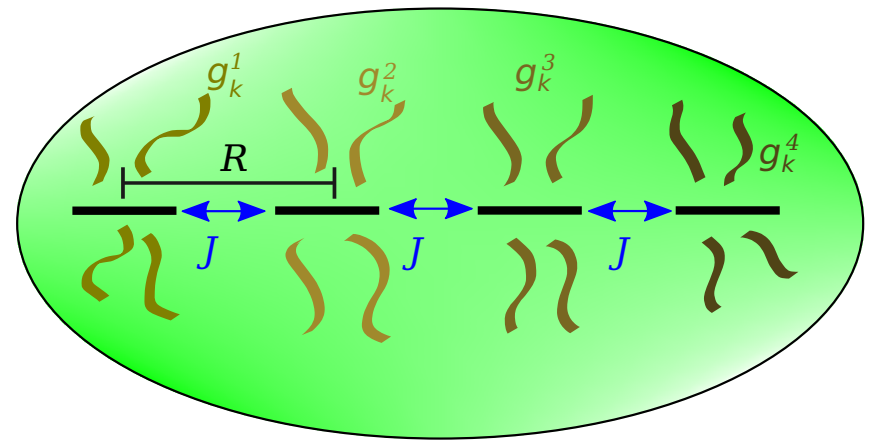

FIG. 3. Schematic diagram of the model under study. A system composed of interacting sites is embedded into a single bosonic environment. Each site couples differently to the environment.

for example, via the use of swap gates for TEBD [26]), thus increasing its computational cost or decreasing its accuracy. Putting all these elements together, in Sec. III we demonstrate regimes of the model where long-time and even periodic communication between the sites is mediated by the environment.

\section{METHODS}

\section{A. Model}

We consider a one-dimensional chain of $N$ sites $\{\alpha\}$ in a common one-dimensional bosonic bath with modes characterized by the wave vectors $k \in\left[-k_{c},+k_{c}\right]$, where $k_{c}$ is the environmental cutoff wave vector. The environmental dispersion relation is given by $\omega_{k}=|k| c$, with $c$ the speed of the phonons in the bath. We restrict ourselves to the singleexcitation subspace of the system described by a Hamiltonian $\hat{H}_{S}$ with nearest-neighbor hopping

$$
\begin{aligned}
\hat{H}= & \hat{H}_{S}+\hat{H}_{E}+\hat{H}_{\mathrm{int}} \\
= & \sum_{\alpha=1}^{N} E_{\alpha}|\alpha\rangle\langle\alpha|+\sum_{\alpha=1}^{N-1} J(|\alpha\rangle\langle\alpha+1|+\text { H.c. }) \\
& +\int_{-k_{c}}^{k_{c}} \omega_{k} \hat{a}_{k}^{\dagger} \hat{a}_{k} d k+\sum_{\alpha}|\alpha\rangle\langle\alpha| \int_{-k_{c}}^{k_{c}}\left(g_{k}^{\alpha} \hat{a}_{k}+\text { H.c. }\right) d k
\end{aligned}
$$

where $\hat{a}_{k}$ is the annihilation operator of a bath mode of wave vector $k, g_{k}^{\alpha}=g_{k} e^{i k r_{\alpha}}$, with $g_{k}=g_{-k} \in \mathbb{R}$, is the coupling strengths between the system and the bath, and $r_{\alpha}$ is the position of the site $\alpha$.

Here the interaction between the excitation and the bath depends explicitly on the position of this excitation on the chain through the phases of the coupling constants $g_{k}^{\alpha}$. We call this type of coupling a plane-wave coupling. A schematic of the model is presented in Fig. 3.

In the rest of the paper we consider a system made of two degenerate sites with an initial state where the system and its environment are decoupled and the bath is empty

$$
|\psi(t=0)\rangle=|S(0)\rangle \bigotimes_{k \in\left[-k_{c}, k_{c}\right]}\left|0_{k}\right\rangle,
$$

where $|S(0)\rangle$ is the initial state of the system and $\left|0_{k}\right\rangle$ represents the vacuum state of the mode $k$ of the bath. Adding extra system sites does not add any complexity to our method, but for simplicity and ease of interpretation of results in this paper we only present results for two system sites. As we will explain in Sec. II B, the empty bath can also be used to effectively describe a Gibbs state at temperature $T$, and so we are able to extend our results to nonzero temperature. Even though the method presented in this paper works for any initial state of the system, in Secs. III and IV the initial state of the system is chosen to be the highest-energy eigenstate (i.e., upper eigenstate) of the system Hamiltonian $\hat{H}_{S}$.

In order to study the time evolution of the system and its bath, we use a numerically exact method based on the implementation of the time-dependent variational principle with a tensor network formulation using a MPS Ansatz for the quantum states [27]. This method requires a discrete representation of the environment in order to write the MPS and to write the Hamiltonian as a MPO.

\section{B. Environment chain mapping}

Instead of sampling $k$ modes of the environment to keep only a discrete set of modes, we use a chain mapping approach that enables us to keep all the relevant bath modes easily and at the same time generate a discrete representation of the environment [22,28,29]. This method consists of using a unitary transformation defined through a family of orthonormal polynomials that transforms a continuous bosonic environment into a semi-infinite chain and is known as the time-evolving density matrix with orthonormal polynomials algorithm.

\section{Zero temperature}

We separate positive and negative wave-vector modes and apply to them two different chain mappings and we note that $\hat{b}_{k} \stackrel{\text { def }}{=} \hat{a}_{-k}$. The bath and interaction Hamiltonians become

$$
\begin{aligned}
\hat{H}_{E}+\hat{H}_{\mathrm{int}}= & \int_{0}^{k_{c}} d k \omega_{k}\left(\hat{a}_{k}^{\dagger} \hat{a}_{k}+\hat{b}_{k}^{\dagger} \hat{b}_{k}\right) \\
& +\sum_{\alpha}|\alpha\rangle\langle\alpha| \int_{0}^{k_{c}} d k g_{k}\left[e^{i k r_{\alpha}}\left(\hat{a}_{k}+\hat{b}_{k}^{\dagger}\right)+\text { H.c. }\right] .
\end{aligned}
$$

We now introduce two unitary transformations

$$
\begin{aligned}
& \hat{a}_{k \geqslant 0}=\sum_{n} U_{n}(k) \hat{c}_{n}, \\
& \hat{b}_{k \geqslant 0}=\sum_{m} V_{m}(k) \hat{d}_{m},
\end{aligned}
$$

where the matrix elements are

$$
U_{n}(k)=V_{n}(k)=g_{k} P_{n}(k),
$$

where $\left\{P_{n}\right\}_{n \in \mathbb{N}}$ are orthonormal polynomials with respect to the measure $\mu(k)=\left|g_{k}^{\alpha}\right|^{2}=g_{k}^{2} \stackrel{\text { def }}{=} J(k)$ (which is the bath spectral density) such that $P_{0}(k)=1$ and

$$
\int_{0}^{k_{c}} P_{n}(k) P_{m}(k) J(k) d k=\delta_{n, m} .
$$

The nature of the polynomials thus depends on the spectral density of the bath. We choose an Ohmic spectral density with a hard cutoff (here at $\left.k_{c}\right) J(k)=2 \alpha k H\left(k_{c}-k\right)$, where $\alpha$ is a 
coupling strength and $H$ the Heaviside step function, as it is neither a trivial (i.e., flat) nor an exotic spectral density that would obscure the effects induced by the spatial correlations. Moreover, this spectral density is commonly used in biological contexts [30]. In that case, the $P_{n}$ are Jacobi polynomials. Another useful property of these polynomials is that they obey a recurrence relation

$$
P_{n}(k)=\left(k-A_{n-1}\right) P_{n-1}(k)+B_{n-1} P_{n-2}(k),
$$

where $A_{n}$ is related to the first moment of $P_{n}$ and $B_{n}$ to the norms of $P_{n}$ and $P_{n-1}$ [22]. This recurrence relation can be used to construct the polynomials with the conditions that $P_{0}(k)=1$ and $P_{-1}(k)=0$. We can then map the bath Hamiltonian using the unitary transformations from Eqs. (5) and (6) to two tight-binding chains with the same on-site energies $\omega_{n}$ and hopping energies $t_{n}$ :

$$
\begin{aligned}
\hat{H}_{E}= & \sum_{n} \omega_{n}\left(\hat{c}_{n}^{\dagger} \hat{c}_{n}+\hat{d}_{n}^{\dagger} \hat{d}_{n}\right) \\
& +t_{n}\left(\hat{c}_{n}^{\dagger} \hat{c}_{n+1}+\hat{c}_{n+1}^{\dagger} \hat{c}_{n}+\hat{d}_{n}^{\dagger} \hat{d}_{n+1}+\hat{d}_{n+1}^{\dagger} \hat{d}_{n}\right) .
\end{aligned}
$$

For the interaction Hamiltonian, we apply the same procedure and make use of Eq. (9) and find that the chains couple to the system with coupling coefficients $\gamma_{n}\left(r_{\alpha}\right)$ and $\gamma_{n}\left(r_{\alpha}\right)^{*}$,

$$
\hat{H}_{\text {int }}=\sum_{\alpha}|\alpha\rangle\langle\alpha| \sum_{n}\left[\gamma_{n}\left(r_{\alpha}\right)\left(\hat{c}_{n}+\hat{d}_{n}^{\dagger}\right)+\text { H.c. }\right]
$$

where

$$
\gamma_{n}\left(r_{\alpha}\right)=\int_{0}^{k_{c}} d k J(k) e^{i k r_{\alpha}} P_{n}(k) .
$$

In preceding works, the TEDOPA resulted in the system being connected only to the first site of the chain. By contrast, here the system is generally coupled to all the sites of the chain, as represented in Fig. 4(a).

\section{Finite temperature}

This chain mapping technique has been extended to describe finite-temperature systems in a statistical mixture as an equivalent zero-temperature state vector under the name thermalized time-evolving density matrix with orthonormal polynomials algorithm [24,31]. It relies on allowing the bath to have negative frequency modes to describe thermal fluctuations and using an alternative bath spectral density that captures the temperature dependence. To identify this new effective spectral density, we put the finite-temperature bath autocorrelation functions $C_{\beta}(r, t)$ for propagating and counterpropagating modes in the form of a zero-temperature autocorrelation $C_{\infty}(r, t)$.

The interaction Hamiltonian in interaction picture is

$$
\begin{aligned}
\hat{H}_{\mathrm{int}}^{I}= & \sum_{\alpha}|\alpha\rangle\langle\alpha| \int_{0}^{k_{c}} d k g_{k}\left(e^{i\left(k r_{\alpha}-\omega_{k} t\right)} \hat{a}_{k}+\text { H.c. }\right) \\
& +\sum_{\alpha}|\alpha\rangle\langle\alpha| \int_{0}^{k_{c}} d k g_{k}\left(e^{-i\left(k r_{\alpha}+\omega_{k} t\right)} \hat{b}_{k}+\text { H.c. }\right) \\
= & \sum_{\alpha}|\alpha\rangle\langle\alpha|\left[\hat{B}_{r_{\alpha}}^{1}(t)+\hat{B}_{r_{\alpha}}^{2}(t)\right] .
\end{aligned}
$$

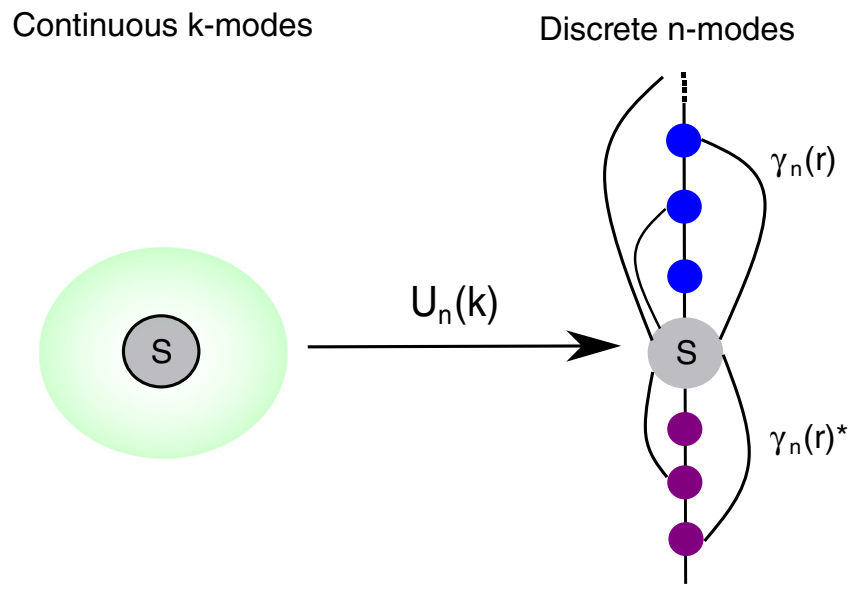

(a)

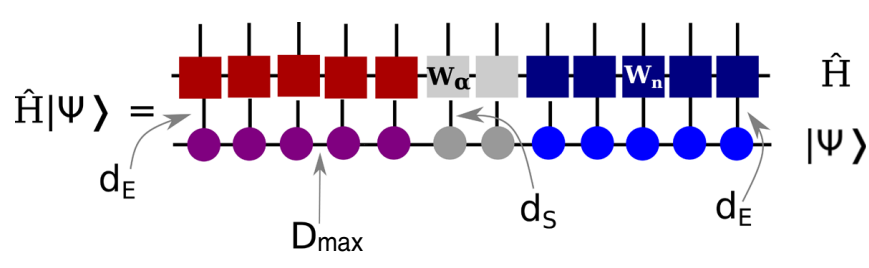

(b)

FIG. 4. (a) The unitary transformation $U_{n}(k)$ transforms a continuous environment of uncoupled $k$ modes to semi-infinite discrete tight-binding chains. (b) Schematic diagram of the MPS representation of the wave function of the system and the chain. The circles represent individual tensors whose rank is given by their number of legs. The open legs correspond to physical Hilbert spaces of dimensions $d_{S}$ for the system and $d_{E}$ for the environment. The horizontal legs are virtual bonds related to the amount of correlation between sites; their maximal dimension is $D_{\max }$. When a leg is shared between two tensors they are contracted, i.e., summed over the corresponding index.

Hence the bath correlation function for the propagating modes is

$$
\begin{aligned}
C_{\beta}\left(r-r^{\prime}, t\right)= & \left\langle\hat{B}_{r}^{1}(t) \hat{B}_{r^{\prime}}^{1}(0)\right\rangle_{B} \\
= & \int_{0}^{k_{c}} d k J\left(\omega_{k}\right)\left\{n_{\beta}\left(\omega_{k}\right) e^{-i\left[k\left(r-r^{\prime}\right)-\omega_{k} t\right]}\right. \\
& \left.+\left[n_{\beta}\left(\omega_{k}\right)+1\right] e^{i\left[k\left(r-r^{\prime}\right)-\omega_{k} t\right]}\right\},
\end{aligned}
$$

where $n_{\beta}\left(\omega_{k}\right)$ is the Bose-Einstein distribution and $\beta=$ $\left(k_{B} T\right)^{-1}$ is the inverse temperature. A more detailed derivation can be found in Appendix A. We could write the corresponding correlation function for the counterpropagating modes which would be the same except for the sign of the wave number $k$. These two correlation functions have, in addition to the usual temperature dependence $\beta$ and time dependence $t$, a spatial dependence $r-r^{\prime}$ originating from the spatial dependence of the coupling coefficients $\left\{g_{k}^{\alpha}\right\}$ between the system and the bosonic bath. Hence these correlation functions contain more information (i.e., about space and time) than the correlation functions usually encountered. For zero temperature, the correlation function reduces to

$$
C_{\infty}\left(r-r^{\prime}, t\right)=\int_{0}^{k_{c}} d k J\left(\omega_{k}\right) e^{i\left(k\left(r-r^{\prime}\right)-\omega_{k} t\right)} .
$$


We want to rewrite $C_{\beta}\left(r-r^{\prime}, t\right)$ in the same form as Eq. (17), i.e., the integral of a spectral density times a planewave phase factor. In other words, we want to find a bath at zero $T$ with a different spectral density but with the same system dynamics as the finite- $T$ bath.

We recast the first term of $C_{\beta}\left(r-r^{\prime}, t\right)$ in Eq. (16) such that the argument of the exponential is the same as the second term by sending $k \rightarrow-k$, allowing for negative frequencies (hence, $\omega_{-k}=-\omega_{k}$ ) and using the identity

$$
n_{\beta}\left(-\omega_{k}\right)=-\left[n_{\beta}\left(\omega_{k}\right)+1\right] \text {. }
$$

With this transformation we have, in a sense, double the number of propagating modes. There are the propagating positive $k$ modes with positive energies and the propagating negative $k$ modes with negative energies (coming from the second term of the correlation function).

Finally, the bath correlation function for propagating modes can be written

$$
C_{\beta}\left(r-r^{\prime}, t\right)=\int_{-k_{c}}^{k_{c}} d k J_{\mathrm{ext}}\left(\omega_{k}\right)\left[n_{\beta}\left(\omega_{k}\right)+1\right] e^{i\left[k\left(r-r^{\prime}\right)-\omega_{k} t\right]},
$$

where $J_{\text {ext }}$ is the spectral density with a domain extended to negative frequencies and antisymmetrized such that $J_{\text {ext }}\left(-\left|\omega_{k}\right|\right)=-J_{\text {ext }}\left(\left|\omega_{k}\right|\right)$. The same procedure can be applied to the counterpropagating modes. We can thus define orthonormal polynomials with the finite-temperature spectral density

$$
J_{\beta}(k)=J_{\text {ext }}\left(\omega_{k}\right)\left[n_{\beta}\left(\omega_{k}\right)+1\right],
$$

which is always positive and continuously differentiable. We define the unitary transformation to chain modes

$$
\begin{array}{ll}
\hat{a}_{k}=\sum_{n} U_{n}^{\beta}(k) \hat{c}_{n} & \text { for } k \in\left[-k_{c},+k_{c}\right], \\
\hat{b}_{k}=\sum_{n} U_{n}^{\beta}(k) \hat{d}_{n} & \text { for } k \in\left[-k_{c},+k_{c}\right],
\end{array}
$$

where $U_{n}^{\beta}(k)=\sqrt{J_{\beta}(k)} P_{n}^{\beta}(k)$ and $P_{n}^{\beta}(k)$ is a polynomial of order $n$ from a family of orthonormal polynomials with respect to the measure $d \mu(k)=J_{\beta}(k) d k$, i.e.,

$$
\int_{-k_{c}}^{k_{c}} P_{n}^{\beta}(k) P_{m}^{\beta}(k) d \mu(k)=\delta_{n, m} .
$$

With this set of orthogonal polynomials, we can map the environment to two tight-binding chains and a coupling coefficient

$$
\gamma_{n}(r)=\int_{-k_{c}}^{k_{c}} d k J_{\beta}\left(\omega_{k}\right) e^{i k r} P_{n}^{\beta}(k)
$$

between the system and the $\hat{a}_{k}$ and $\hat{b}_{k}^{\dagger}$ operators.

\section{Hamiltonian MPO formulation}

To construct the MPO representation of a Hamiltonian $\hat{H}$ which is made of a sum of local terms, we use a method based on the recurrence relation presented in [32]. To define the $k$ th tensor of the MPO, we have to decompose the Hamiltonian into a part that describes what happens before the bond $k$ (which is the bond connecting site $k$ and site $k+1) \hat{H}_{k-1}^{L}$, after the bond $k \hat{H}_{k+1}^{R}$, and at bond $k \sum_{a} \hat{h}_{k a}^{L} \otimes \hat{h}_{k a}^{R}$,

$$
\hat{H}=\hat{H}_{k-1}^{L} \otimes \hat{\mathbb{1}}_{k}^{R}+\hat{\mathbb{1}}_{k}^{L} \otimes \hat{H}_{k+1}^{R}+\sum_{a} \hat{h}_{k a}^{L} \otimes \hat{h}_{k a}^{R},
$$

where $\hat{\mathbb{1}}_{k}^{R}=\underbrace{\hat{\mathbb{1}} \otimes \cdots \otimes \hat{\mathbb{1}}}_{N-k+1 \text { times }}$ and $\hat{\mathbb{1}}_{k}^{L}=\underbrace{\hat{\mathbb{1}} \otimes \cdots \otimes \hat{\mathbb{1}}}_{k \text { times }}$. The last term of Eq. (25) is an interaction Hamiltonian between the part of the system on the left of bond $k$ and the one on the right of bond $k$. Hence $\hat{h}_{k a}^{L}$ contains an operator defined on the left of $k$ and $\hat{h}_{k a}^{R}$ an operator defined on the right of $k$ (e.g., for an $X Y Z$ Hamiltonian with nearest-neighbor couplings, we could have $\hat{h}_{k a}^{L}=J_{a} \hat{S}_{k}^{a}$ and $\hat{h}_{k a}^{R}=\hat{S}_{k+1}^{a}$ with $\left.a \in\{x, y, z\}\right)$. A recurrence relation between the right parts of the Hamiltonian at two consecutive sites can be defined

$$
\left(\begin{array}{c}
\hat{H}_{k}^{R} \\
\hat{h}_{k}^{R} \\
\hat{\mathbb{1}}_{k}^{R}
\end{array}\right)=W_{k+1}\left(\begin{array}{c}
\hat{H}_{k+1}^{R} \\
\hat{h}_{k+1}^{R} \\
\hat{\mathbb{1}}_{k+1}^{R}
\end{array}\right),
$$

with the matrices $W_{k}$ defining the Hamiltonian MPO

$$
\hat{H}=\sum_{\sigma, \sigma^{\prime}, w} W_{1 w_{1}}^{\sigma_{1} \sigma_{1}^{\prime}} W_{2 w_{1} w_{2}}^{\sigma_{2} \sigma_{2}^{\prime}} \cdots W_{N w_{N-1}}^{\sigma_{N} \sigma_{N}^{\prime}}\left|\sigma_{1} \cdots \sigma_{N}\right\rangle\left\langle\sigma_{1}^{\prime} \cdots \sigma_{N}^{\prime}\right| .
$$

In Eq. (27) the $\sigma$ and $\sigma^{\prime}$ indices refer to the local Hilbert spaces of the different parts of the system (i.e., site and chain modes) whereas the $w$ indices relate to virtual bonds between the different parts of the system. The bath modes will be considered as extra sites where different kinds of excitations [which couple to the excitation existing on the sites with the $\gamma_{n}^{\alpha} \stackrel{\text { def }}{=} \gamma_{n}\left(r_{\alpha}\right)$ coefficients] can exist. We introduce a new set of commuting operators $\left\{\hat{f}_{\alpha}\right\}$ such that $|\alpha+1\rangle\langle\alpha|=\hat{f}_{\alpha+1}^{\dagger} \hat{f}_{\alpha}$. Figure 4(b) shows a schematic diagram of the MPO and how it contracts with a MPS. The on-site tensor has a bond dimension $D=2(\alpha+2)$ for the $\alpha$ th site and a physical dimension (dimension of the local Hilbert space) $d_{S}=2$,

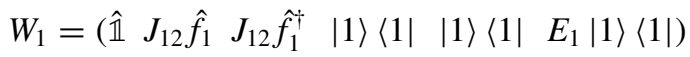

and

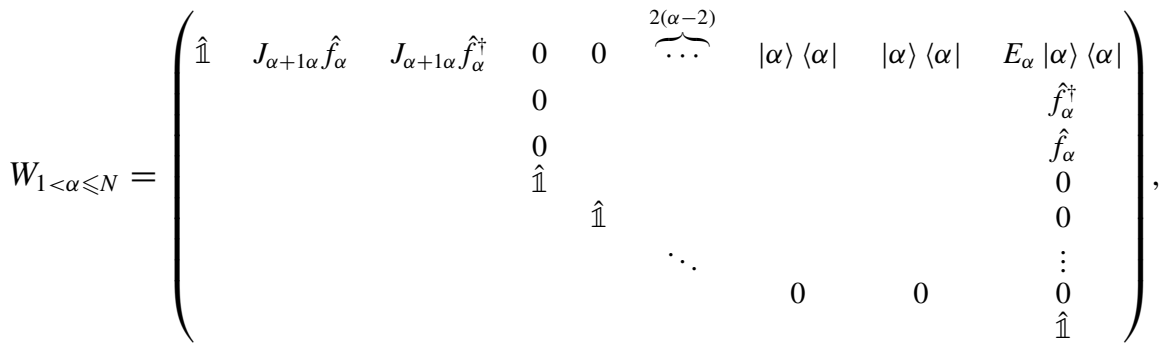


with $J_{N+1 N}=0$ for the last system tensor. The chain on-site tensor has a similar structure, but with a constant bond dimension for each mode. The on-site tensor has a bond dimension $D=2(N+2)$ and, in principle, a physical dimension $d=\infty$ that we truncate to a value $d_{E}$ in our numerical treatment. The number of sites of the two semi-infinite chains is also truncated at large enough values $N_{m}$ and $N_{m}^{\prime}$ such that an excitation on the chain does not have the possibility to reach the end of the chain during the time evolution

$$
W_{1 \leqslant n \leqslant N_{m}}=\left(\begin{array}{cccccccc}
\hat{\mathbb{1}} & t_{n} \hat{c}_{n}^{\dagger} & t_{n} \hat{c}_{n} & 0 & 0 & \cdots & 0 & \omega_{n} \hat{c}_{n}^{\dagger} \hat{c}_{n} \\
& & & 0 & & & & \hat{c}_{n} \\
& & & 0 & & & & \hat{c}_{n}^{\dagger} \\
& & & \hat{\mathbb{1}} & & & & \gamma_{n}^{1} \hat{c}_{n} \\
& & & \hat{\mathbb{1}} & & & \gamma_{n}^{1 *} \hat{c}_{n}^{\dagger} \\
& & & & \ddots & & \vdots \\
& & & & & \hat{\mathbb{1}} & \gamma_{n}^{N *} \hat{c}_{n}^{\dagger} \\
& & & & & & \\
& & & & & & & \\
& & & & & & &
\end{array}\right.
$$

with $t_{N_{m}}=0$. The second chain's tensors are identical with $\hat{d}_{n^{\prime}}$ and $\gamma_{n^{\prime}}(r) \hat{d}_{n^{\prime}}^{\dagger}$ instead of $\hat{c}_{n}$ and $\gamma_{n}(r) \hat{c}_{n}$, where $n^{\prime}$ corresponds to the mirror site on the other chain. The last tensor is

$$
W_{N_{m}^{\prime}}=\left(\begin{array}{c}
\omega_{N_{m}^{\prime}} \hat{d}_{N_{m}^{\prime}}^{\dagger} \hat{d}_{N_{m}^{\prime}} \\
\hat{d}_{N_{m}^{\prime}}^{\prime} \\
\hat{d}_{N_{m}^{\prime}}^{\dagger} \\
\gamma_{N_{m}^{\prime}}^{*} \hat{d}_{n^{\prime}}^{\dagger} \\
\gamma_{N_{m}^{\prime}}^{1 *} \hat{d}_{n^{\prime}} \\
\vdots \\
\gamma_{N_{m}^{\prime}}^{N *} \hat{d}_{n^{\prime}} \\
\hat{\mathbb{1}}^{\prime}
\end{array}\right)
$$

One might notice that the chain site tensors have a bond dimension $D$ that is fixed by the number of sites in the system $N$. This means that having a large environment only increases the number of individual tensors one needs but not their size. This result is central for the tractability of this approach. The identity operators present on the diagonals carry out along the chain the long-range coupling coefficients such that they are associated with the corresponding system site. Hence, they allow a local representation of the Hamiltonian as a MPO even though the interactions are long range across the chain.

To illustrate how the Hamiltonian is recovered from these tensors, we perform the calculation in the case where there is only one site in the system and two modes on a unique chain. In that case there are only three tensors

$$
\begin{aligned}
& W_{1}=\left(\hat{\mathbb{1}} \quad|1\rangle\langle 1|\quad| 1\rangle\left\langle 1\left|\quad E_{1}\right| 1\right\rangle\langle 1|\right), \\
& W_{2}=\left(\begin{array}{cccccc}
\hat{\mathbb{1}} & t_{1} \hat{c}_{1}^{\dagger} & t_{1} \hat{c}_{1} & 0 & 0 & \omega_{1} t_{1} \hat{c}_{1}^{\dagger} \hat{c}_{1} \\
0 & 0 & 0 & \hat{\mathbb{1}} & 0 & \gamma_{1}^{1} \hat{c}_{1} \\
0 & 0 & 0 & 0 & \hat{\mathbb{1}} & \gamma_{1}^{1 *} \hat{c}_{1}^{\dagger} \\
0 & 0 & 0 & 0 & 0 & \hat{\mathbb{1}}^{1}
\end{array}\right),
\end{aligned}
$$

$$
W_{3}=\left(\begin{array}{c}
\omega_{2} \hat{c}_{2}^{\dagger} \hat{c}_{2} \\
\hat{c}_{2} \\
\hat{c}_{2}^{\dagger} \\
\gamma_{2}^{1} \hat{c}_{2} \\
\gamma_{2}^{1 *} \hat{c}_{2}^{\dagger} \\
\hat{\mathbb{1}}
\end{array}\right)
$$

The contraction of $W_{2}$ and $W_{3}$ gives a $5 \times 1$ tensor with the same shape as $W_{3}$ and the transpose of the shape of $W_{1}$,

$$
W_{2} \cdot W_{3}=\left(\begin{array}{c}
\omega_{2} \hat{c}_{2}^{\dagger} \hat{c}_{2}+t_{1}\left(\hat{c}_{1}^{\dagger} \hat{c}_{2}+\hat{c}_{1} \hat{c}_{2}^{\dagger}\right)+\omega_{1} \hat{c}_{1}^{\dagger} \hat{c}_{1} \\
\gamma_{2}^{1} \hat{c}_{2}+\gamma_{1}^{1} \hat{c}_{1} \\
\gamma_{2}^{1 *} \hat{c}_{2}^{\dagger}+\gamma_{1}^{1 *} \hat{c}_{1}^{\dagger} \\
\hat{\mathbb{1}}^{\prime}
\end{array}\right) .
$$

Further contraction with $W_{1}$ gives a scalar corresponding to the Hamiltonian

$$
\begin{aligned}
W_{1} \cdot W_{2} \cdot W_{3}= & \omega_{2} \hat{c}_{2}^{\dagger} \hat{c}_{2}+t_{1}\left(\hat{c}_{1}^{\dagger} \hat{c}_{2}+\hat{c}_{1} \hat{c}_{2}^{\dagger}\right)+\omega_{1} \hat{c}_{1}^{\dagger} \hat{c}_{1} \\
& +\gamma_{2}^{1}|1\rangle\left\langle 1\left|\hat{c}_{2}+\gamma_{1}^{1}\right| 1\right\rangle\left\langle 1\left|\hat{c}_{1}+\gamma_{2}^{1}\right| 1\right\rangle\langle 1| \hat{c}_{2} \\
& +\gamma_{1}^{1 *}|1\rangle\left\langle 1\left|\hat{c}_{1}^{\dagger}+\gamma_{2}^{1 *}\right| 1\right\rangle\langle 1| \hat{c}_{2}^{\dagger} \\
& +E_{1}|1\rangle\langle 1| \\
& W_{1} \cdot W_{2} \cdot W_{3}=\hat{H}
\end{aligned}
$$

\section{ZERO TEMPERATURE}

\section{A. Couplings}

Because of the dependence of the system-bath coupling strengths on the spatial configuration of the system, the system-chain couplings are long range. In the cases presented in previous works [24,31] the system only coupled to the first site of the semi-infinite chain. The system could thus only inject excitation at one end of the chain, which then would propagate according only to the tight-binding interactions along the chain. In the present case, the system-chain couplings are long range and thus the system can create excitations on different regions of the chain. Absolute values of the system-chain coupling for zero temperature are shown in Fig. 5 for an Ohmic spectral density.

The first site of the system couples only to the first site of the chain. However, the other sites couple to a range of modes with a maximum strength for the mode $n \sim R / 2 c$, with $R$ the distance between the considered system's site and the first system's site in units of $k_{c}^{-1}$.

We can also see in Fig. 5 that the amplitude of the coupling before the peak decreases with the position of the peak. Said differently, the larger the distance is between the two sites, the less the second site interacts with the beginning of the chain. Thus, we can expect that for infinite separation when $R \rightarrow \infty$ this system will behave like a spin-boson model (SBM). This limit is looked at in Appendix C.

Looking at the opposite limit, when the separation between the two system sites vanishes, Eq. (2) tells us that the system completely decouples from the environment. Because each site in the system couples mostly to a specific region of the chain, we call our model correlated environment in contrast 


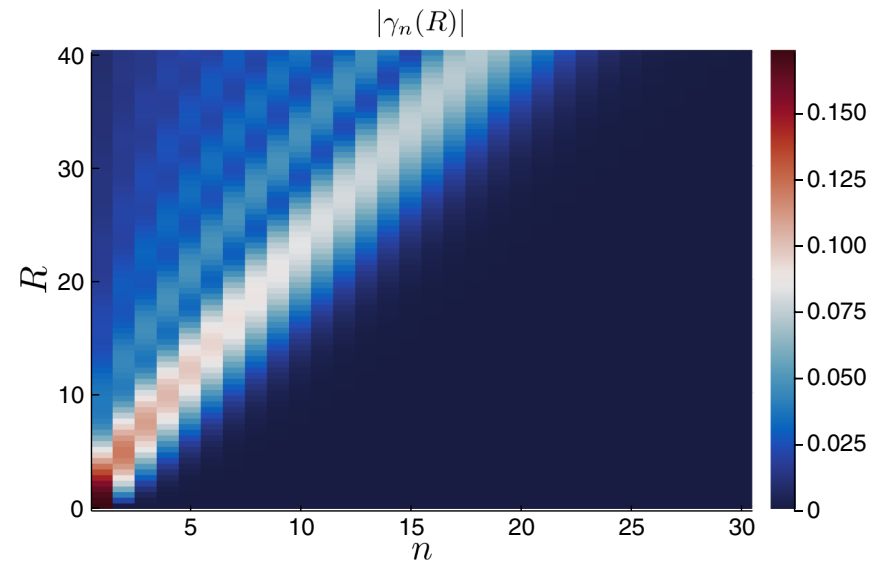

FIG. 5. Absolute value of the system-chain coupling constants, for a bosonic bath with a hard cutoff Ohmic spectral density, as a function of the chain modes $n$ and the site separation $R$. Note that the main peak is centered around $R / 2 c$. Here $\alpha=0.12, c=1$, and $k_{c}=1$.

with the cases where the system couples only to the first site of the chain.

\section{B. Non-Markovian recurrences and bath feedback}

At zero temperature, the dynamics of the two-level system in a bosonic environment is well known and described by the SBM [1]. In the system's eigenbasis, the population of the upper state (high-energy state) should spontaneously decay to the lower state on a timescale given by the intensity of the coupling between the system and the bath. Figure 6(b) shows the evolution of the eigenpopulations with an initial state of the system being the upper eigenstate. We clearly see that the upper level population decays as expected until $\omega_{c} t \approx R / c$ when a revival happens. This revival corresponds to an increased localization of the excitation on the second

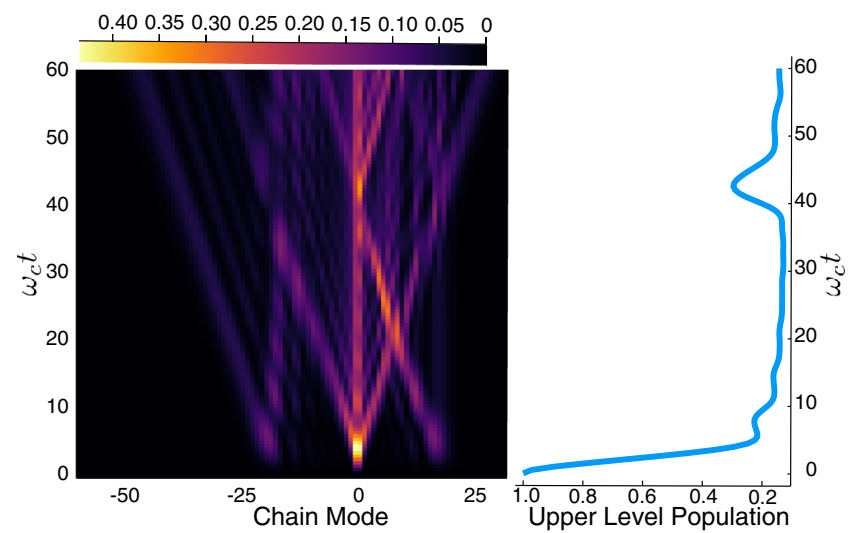

(a)

(b)

FIG. 6. System and bath dynamics. (a) Heatmap of the chain occupation in time showing the propagation of bath excitations along the chains. (b) Upper eigenstate population. An eigenstate revival and a site localization are associated with a chain excitation reaching the beginning of the chain. The separation between the two sites is $R=40$, their coupling is $J=0.25$, the speed of sound is $c=1$, $\alpha=0.12$, and $k_{c}=1$. site of the system after following an evolution in a spatial superposition. With the same conditions, the SBM exhibits the same dynamics except for the revival. However, we note that the two-site case presented here can be mapped to a SBM with an effective spectral density depending on $R$ (see Appendix B), but this property is "accidental" and does not generalize to larger systems.

The study of the bath in the chain representation allows us to have a spatial interpretation of the interaction between the system and its environment as the maximum coupling between a system's site and the chain is localized around $n=R / 2 c$. Figure 6(a) shows a heatmap of the occupation of the modes of the chains as a function of time. The positive and negative chain modes each correspond to one of the two chains necessary to take into account propagating and counterpropagating $k$ modes. The corresponding initial system state is an excitation delocalized on the two sites with a separation $R=40$

We can see that the chain modes around $n= \pm R / 2 c=$ \pm 20 get populated first and that the corresponding bath's excitations then propagate on the chains. At $\omega_{c} t \approx 20$ an excitation propagating from the mode $n=0$ coupled mostly to the first site and an excitation propagating from the mode $n=20$ constructively interfere around $n=10$. The former continues to propagate on the chain and traces a ray in the diagram. The latter reaches $n=0$ at $\omega_{c} t \approx 40$ and is reflected. We can see from this diagram that revivals happen when the excitation emitted along the chain by one site reaches the part of the chain interacting with the other site. We thus have a feedback effect of the environment on the system.

The dynamics of the chain with negative modes is not the reflection of the dynamics of the chain with positive modes. Indeed, the negative chain modes correspond to the propagating $k$ modes; hence the excitations created by the second site move away from the origin of the chain (which is coupled to the first site). On the contrary, bath's excitations created by the second site on the positive modes chain correspond to the counterpropagating $k$ modes and move toward the origin of the chain. On both chains the excitations created by the first system site propagate toward the end of the chain as they move away in real space from the first site. This explains the apparent "asymmetry" between the two chains.

The dynamics of the system, all other parameters being the same, only depends on the ratio $R / c$. This is also true for the chain dynamics, for example, the $(R=40, c=1)$ and $(R=$ $20, c=0.5$ ) cases have the same heatmaps. This was expected as the system's sites couple in both cases to the same parts of the chain and the bath's excitations travel on the chain at the same speed.

Increasing the propagation speed of the bath excitations, we can generate several revivals with something like an echo between the two sites, as shown in Fig. 7, where revivals with decreasing amplitudes can be observed with a periodicity of $R / c$. All the parameters are the same as in Fig. 6 except the speed of the bath's excitations that has been doubled.

Figure 7(a) shows the heatmap of the chains for the same parameters as in Fig. 6 except the speed of bosonic excitation $c$ which is doubled. We note that even though $c$ is doubled, the speed of the excitation on the chain remains the same as the rays in both Figs. 6 and 7 travel the same 


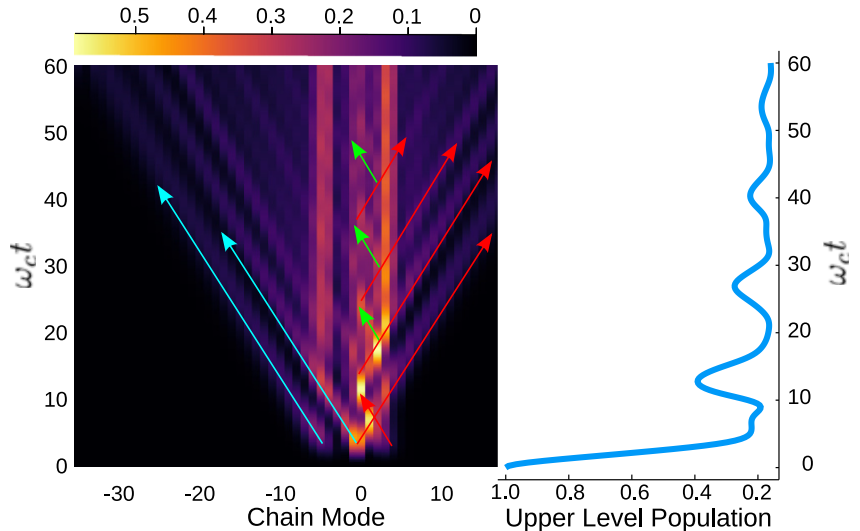

(a)

(b)

FIG. 7. (a) Heatmap of the chain occupation in time showing the propagation of bath excitations along the chains. Arrows have been added to represent the trajectories of the chains' excitations. (An unannotated figure is given in Appendix D.) (b) System eigenstate population for an initial state in the upper eigenstate. The separation between the two sites is $R=20$, their coupling is $J=0.25$, the speed of sound is $c=2, \alpha=0.12$, and $k_{c}=1$. We can definitely see a revival of population at a time consistent with the amount of time needed for a bosonic excitation to travel into the bath from one system's site to the other.

distance along the chain in the same time. The propagation speed on the chain is independent of the coupling strength $\alpha$ or the bosonic excitation speed $c$. The propagation speed on the chain depends on the asymptotic hopping energy between the sites of the chain, which depends on the cutoff frequency $\omega_{c}$ of the spectral density, which is here held constant [22]. However, for a fixed separation $R$, for $c=2$ the modes for which the coupling between the chain and the second system site is maximal are twice as close to the origin as the ones for $c=1$ (as seen in Sec. III A). Hence, for a given $R$, it takes half the time for an excitation to travel from the second to the first system site for $c=2$ than for $c=1$. The four revivals of eigenpopulation that we see in Fig. 7 correspond to the four rays on the positive chain that come from internal reflections of the initial chain excitation highlighted with arrows. These rays correspond to transmitted parts of the bath's excitations bouncing back and forth between the two system sites.

To see the influence of the coupling strength $\alpha$ between the system and the bath, we varied it while keeping a fixed separation $R$ between the system's sites and a fixed speed of the bosonic excitation $c$. These results are presented in Fig. 8, where we can see that increasing the coupling strength sharpens the revivals and brings their peaks closer to $\omega_{c} t \approx R / c$. The amplitude of the revivals decreases with the increase of the upper level population prior to the revival.

Figure 9 shows the coherence between the two sites in the case described by Fig. 6 where the initial state of the system is the upper eigenstate. For a degenerate two-level system, the coherences are proportional to the upper eigenstate population. This means that the revivals coincide with a decrease of coherences in absolute value. A decrease of coherences is hence associated with relocalization.

Another way to show that this revival of eigenpopulation (relocalization) is an incoherent mechanism is to look at the

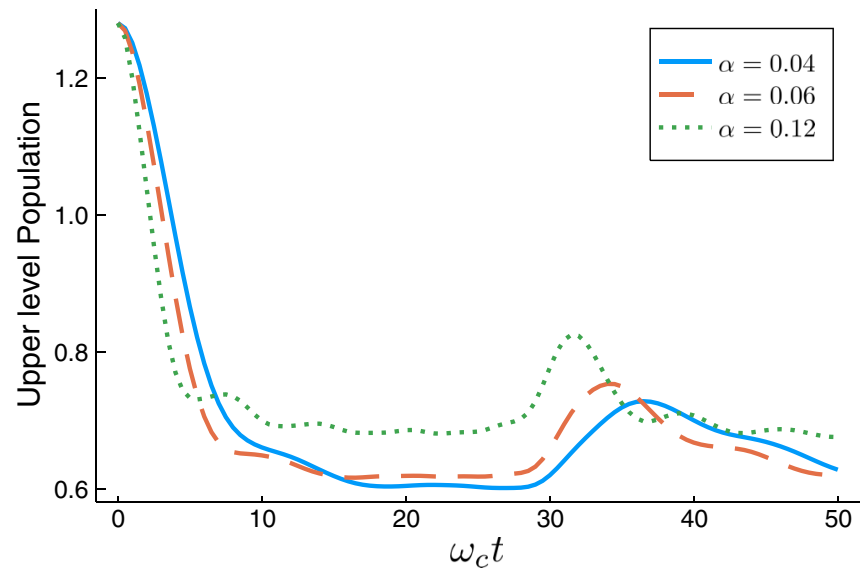

FIG. 8. Comparison of the dynamics of the upper eigenstate at zero temperature for different values of the coupling to the bath $\alpha$. As the coupling increases, the revivals become sharper. The other parameters are held constant at $R=30, k_{c}=1, c=1$, and $J=0.25$.

evolution of the purity $\lambda=\operatorname{tr}\left(\rho_{S}^{2}\right)$ of the system state. The purity measures how close the state is to a pure state: For $\lambda=1$, the state is a pure state and for $\lambda=0.5$ the state of a two-level system is a maximal statistical mixture. Figure 10 presents the evolution of the purity and clearly shows that revivals are associated with an increase of mixedness of the system's state.

Hence the mechanism behind the revivals can be seen as a partial measurement by the environment on the system's sites that, as a consequence, relocalizes the system's excitation. As the purity is a first-order approximation of (one minus) the von Neumann entropy, the decreasing purity at the time of a revival can be seen as an increasing entanglement between the system and its environment. This analysis is consistent with what has been previously said and can be further understood by considering the chain sites that couple the most to the system sites as fragments of the environment that can inform us about the system populations [33]. Indeed, knowing when the chain modes around $n=20$, in the case where $R=40$ and

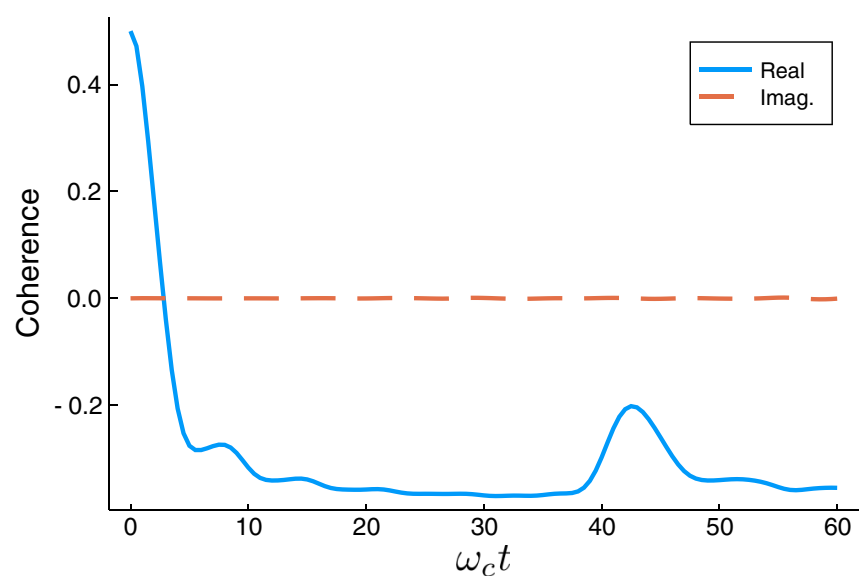

FIG. 9. Real and imaginary parts of the coherence between the two system sites. The real part is proportional to the upper eigenstate population; hence the revival coincides with a sudden loss of coherence. 


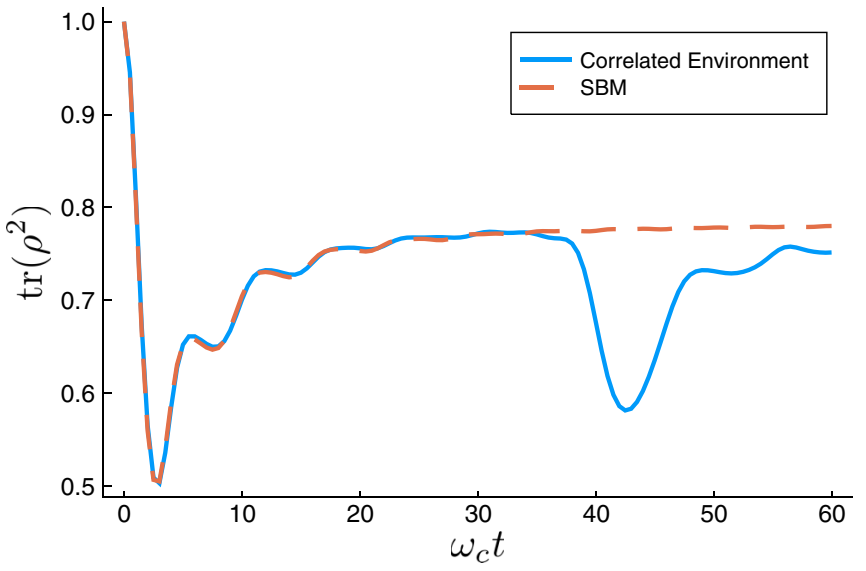

FIG. 10. Purity $\operatorname{tr}\left(\rho_{S}^{2}\right)$ of the system. The revival corresponds to a loss of purity.

$c=1$, have a gain in population enables us to affirm that there will be a revival at this same time.

\section{FINITE TEMPERATURE}

\section{A. Couplings}

The finite-temperature coupling constants between the system and the chain keep broadly the same form as the zerotemperature ones. An example profile for several different system site separations is displayed in Fig. 11. The differences are that the amplitudes increase with temperature and the peak value is no longer centered around the mode $n=R / 2 c$ but rather $n=R / c$. For $\beta=0.5$ the amplitude of the coupling is doubled compared to the zero-temperature case. We also note that the tail before the peak presents more oscillations than the zero-temperature one which is smoother. The change in the coupling profile as a function of temperature is shown in Fig. 12. For high and moderately high temperatures, the couplings decrease in amplitude as $\beta$ increases but are still centered around $n \approx R / c$. For high values of $\beta$, the amplitude

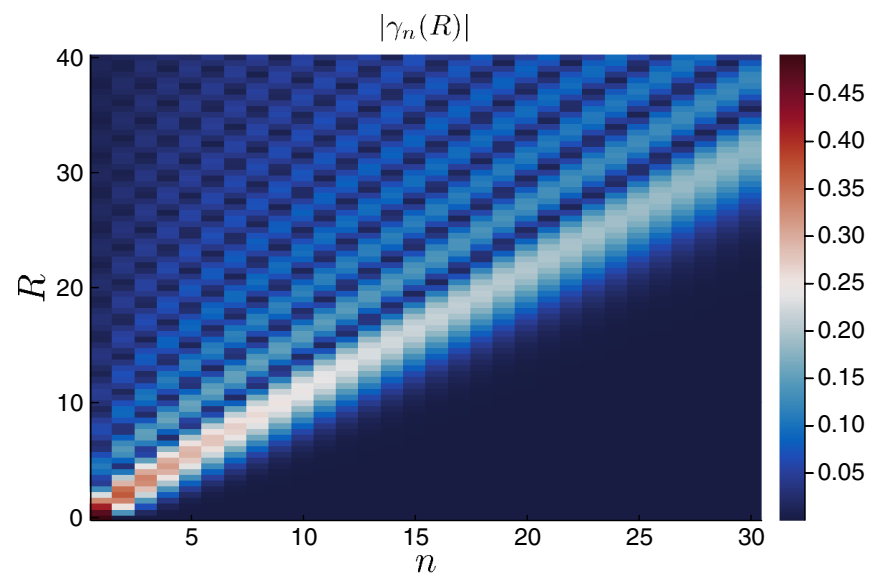

FIG. 11. Absolute value of the system-chain coupling constants at finite temperature, for a bosonic bath with a hard cutoff Ohmic spectral density, as a function of the chain modes $n$ and the site separations $R$. The peaks are centered around $n=R / c$. Here $\alpha=0.12$, $\beta=0.5, c=1$, and $k_{c}=1$.

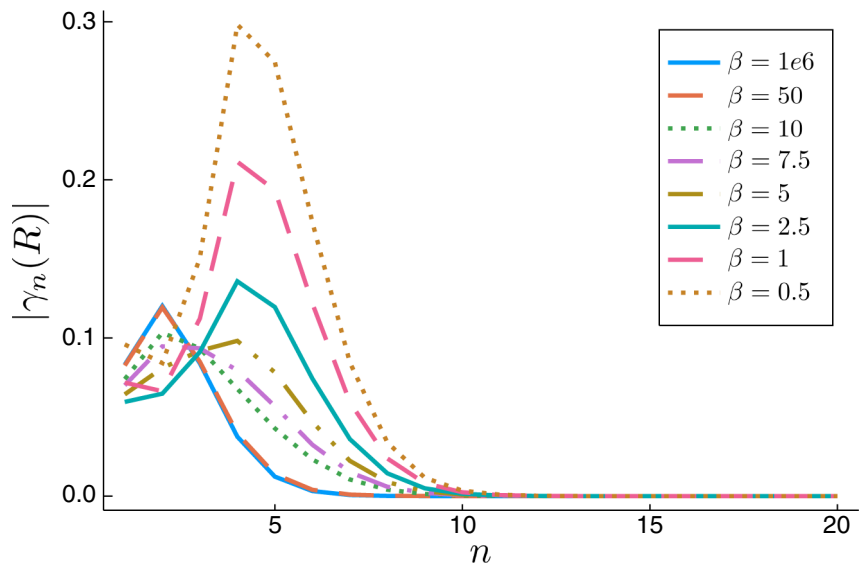

FIG. 12. Absolute value of the system-chain coupling constants at finite temperature, for a bosonic bath with a hard cutoff Ohmic spectral density, as a function of the chain mode number $n$ for a fixed $R=5$ and several temperatures $\left(\alpha=0.12\right.$ and $\left.k_{c}=1\right)$.

stays constant but the maximum swaps to $n \approx R / 2 c$ as we recover the zero-temperature value.

\section{B. Non-Markovian recurrences and bath feedback}

Using the method presented in Sec. II B 2, we also investigate the finite-temperature dynamics of the system. For a large range of values of $\beta$, the system's dynamics stay qualitatively the same except that the steady-state population is increased because of thermal fluctuations, as we can see for $\beta=5$ in Fig. 13.

The peak of the coupling is at $n=R / c$ and not $R / 2 c$ as in the zero-temperature case, but the propagation speed along the chain is doubled because the support of the extended spectral density is twice as large as the support of the zero-temperature spectral density [31]. Figure 13(a) shows the time-frequency diagram for finite temperature for the inverse temperature $\beta=$ 5 and a separation $R=30$. For this intermediate temperature, the chain excitation propagates ballistically in way similar

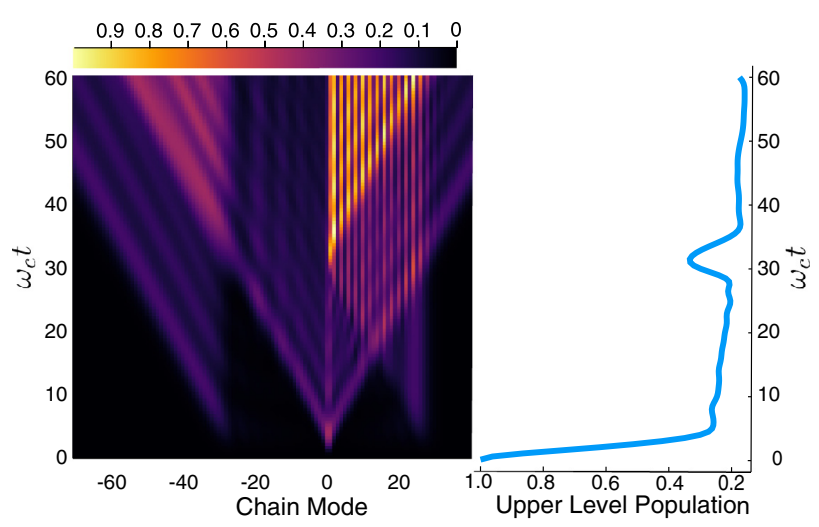

(a)

(b)

FIG. 13. (a) Heatmap of the chain occupation in time showing the propagation of bath excitations along the chains. (b) Upper eigenstate population. The separation between the two sites is $R=30$, the speed of sound is $c=1$, the inverse temperature $\beta=5$, and $\alpha=0.12$. 


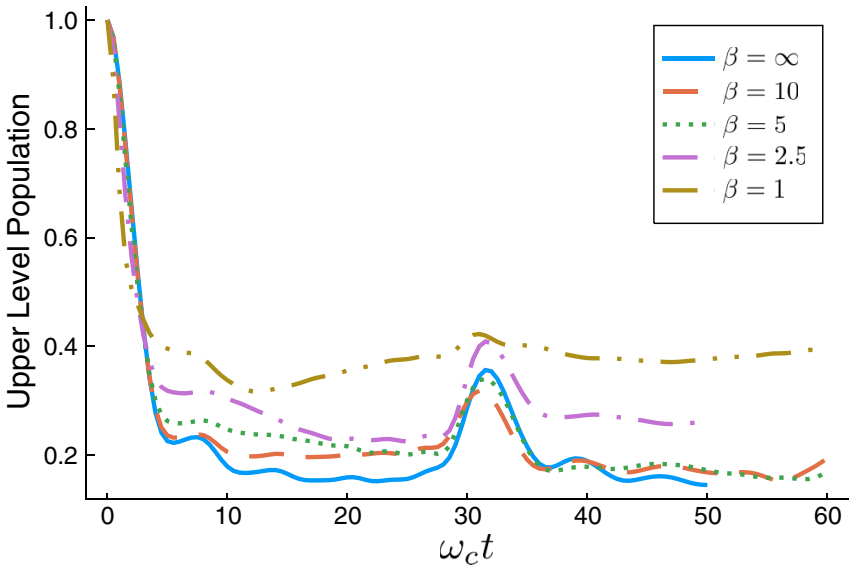

FIG. 14. Upper eigenstate populations for $R=30, \omega_{c}=1, c=$ $1, \omega_{0}=0.25, \alpha=0.12$, and several values of the inverse temperature $\beta$.

to the zero-temperature case, except that modes are more populated due to thermal fluctuations. Wave packets emitted from the origin of the chain and the part coupled to the second site interfere when they meet. Hence, we see interference fringes appear when excitations with different phases come together. As in the finite-temperature case, when excitations reach the origin of the chain they give rise to a revival of the eigenstate population.

Figure 14 shows the upper eigenstate population for increasing values of the temperature. The revivals are still present for moderate temperatures such as $\beta=5$ but they become barely noticeable for high temperature, as we can also see in Fig. 15. Between $\beta=5$ and $\beta=1$ the dynamics of the chains' modes are the same but the populations are increased by a factor $\sim 5$. This increased population is a direct consequence of the thermal population. We can see, in Fig. 14, that the amplitude of the revival seems to be related to the depth of the plateau reached before $\omega_{c} t \approx R / c$. Hence, as the eigenpopulation in this region gets closer to a half, the revival is suppressed.

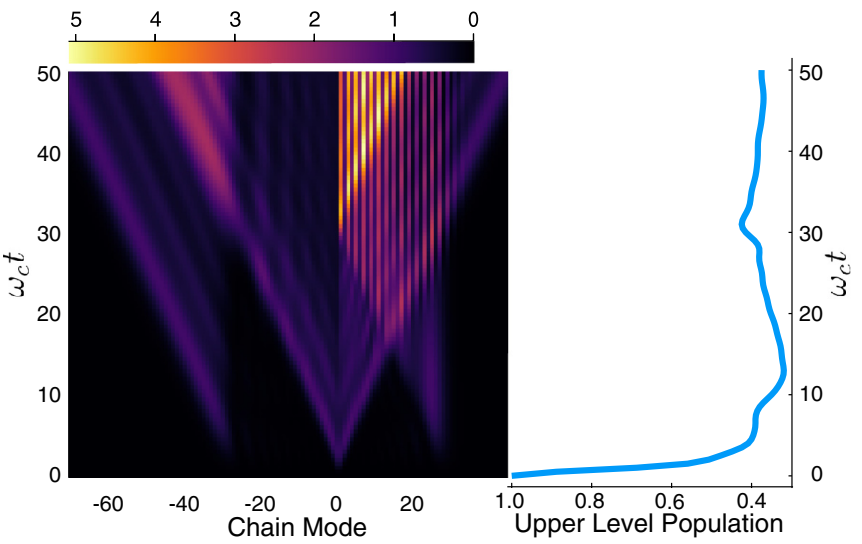

(a)

(b)

FIG. 15. (a) Heatmap of the chain occupation in time showing the propagation of bath excitations along the chains. (b) Upper eigenstate population. For high temperature the revival is less pronounced. The separation between the two sites is $R=30$, the speed of sound is $c=1$, the inverse temperature $\beta=1$, and $\alpha=0.12$.
For higher temperature, as in Fig. 15, the behavior of the chain is akin to the one we could see for a SBM with an Ohmic spectral density [34] but duplicated on the chain. As they propagate on the chain, excitations leave a trail of populated modes behind them that corresponds to the cones we can see in the figure.

\section{CONCLUSION}

Motivated by the ability of biological nanostructures to coordinate (opto)electronic processes through the relaying of environmental (structural) signal motions, we have presented a numerically exact exploration of a model that can describe these highly non-Markovian effects. To do so, we have extended the standard thermalized TEDOPA techniques, in the 1TDVP formulation, to treat the long-range chain couplings that encode information about spatial correlations. In doing so, we have proved that for system-bath problems with spatially correlated interactions, the Hamiltonian matrix product operator will always have a bond dimension proportional to the number of system states, regardless of the range of the interactions. Provided that, as in most models of open systems, the environment is noninteracting, this allows the tensor network approach to be a computationally powerful method for exploring multisite dynamics where nonMarkovian environmental feedback could lead to functionally relevant nonequilibrium states and/or transient effects that could materially alter the outcome of a process, if a certain set of events precedes it.

In our exploration of this aspect of highly structured nanoscale dissipation, we have shown, with a model composed of two sites, that one of the simplest conceptual forms of correlated environments (plane waves in one dimension) supports strong spatiotemporal feedback effects that introduce new timescales into the dissipative dynamics and show clear signs of having stored information about the early-time motion, i.e., after sharp decays, we found sharp revivals. Moreover, we have also found that periodic behavior with $T=R / c$ can also be obtained in which each revival acts as a generator of subsequent revivals, leading to periodic, but highly anharmonic, energy exchange between the system states. Finally, we have shown that finite temperatures tend to broaden and suppress these revival effects, although they visibly persist for temperatures up to the system energy gap.

These results encouragingly point to the idea that suitably tailored environments could be coupled to electronic processes in order to produce well-defined functional effects at later times and in distant places in the structure. To explore this in more detail requires the inclusion of larger, multicomponent systems, and this is something we have shown could be done with the present method. However, in the majority of nanostructures, biological or otherwise, the one-dimensional plane-wave environment is likely to be an oversimplification. It would therefore be of future interest to consider different kinds of relationship between mode frequencies and spatial correlation in the system-bath interactions, such as those that can be extracted by molecular dynamics simulations of proteins [35,36], normal mode analysis [37,38], or coarse-grained methods that access the slow large-amplitude motions of complex structures $[39,40]$. Given that the present method works 
with arbitrarily structured spectral functions and can handle long-range system-environment interactions in the chain or tree tensor representations of the problem, we hope that this work will encourage further examination of the no doubt rich functional phenomenology of spatially correlated open quantum systems.

\section{ACKNOWLEDGMENTS}

T.L., A.C., and B.W.L. thank the Defence Science and Technology Laboratory and Direction Générale de l'Armement for support through the Anglo-French Ph.D. scheme. A.D. acknowledges support from the École Doctorale 564 Physique en Île-de-France. D.G. acknowledges studentship funding from EPSRC (Grant No. EP/L015110/1). B.W.L. acknowledges support from EPSRC Grant No. EP/T014032/1 T.L. acknowledges to be part of the École Doctorale 564 Physique en Île-de-France and the Centre for Doctoral Training in Quantum Materials.

\section{APPENDIX A: CORRELATION FUNCTION}

For a bath operator of the form

$$
\hat{B}_{r}(t)=\int d k\left(g_{k} e^{i k r} e^{-i \omega_{k} t} \hat{a}_{k}+\text { H.c. }\right),
$$

as in Eq. (2), and a bath in a Gibbs state

$$
\hat{\rho}_{B}=\frac{\exp \left(-\beta \hat{H}_{B}\right)}{Z},
$$

where $Z$ is the partition function, the bath correlation function is

$$
\begin{gathered}
C_{\beta}\left(r-r^{\prime}, t-t^{\prime}\right) \stackrel{\operatorname{def}}{=} \operatorname{tr}_{B}\left[\hat{B}_{r}(t) \hat{B}_{r^{\prime}}\left(t^{\prime}\right) \hat{\rho}_{B}\right], \\
C_{\beta}\left(r-r^{\prime}, t-t^{\prime}\right) \\
=\int d k \int d q\left(g_{k}^{r *} g_{q}^{r^{\prime}} e^{i \omega_{k} t-i \omega_{q} t} \operatorname{tr}\left(\hat{a}_{k}^{\dagger} \hat{a}_{q} \hat{\rho}_{B}\right)\right. \\
\left.+g_{k}^{\alpha} g_{q}^{\beta *} e^{i \omega_{q} t^{\prime}-i \omega_{k} t} \operatorname{tr}\left\{\left[\hat{a}_{k}^{\dagger} \hat{a}_{q}+\delta(k-q)\right] \hat{\rho}_{B}\right\}\right), \\
C_{\beta}\left(r-r^{\prime}, t-t^{\prime}\right) \\
=\int d k \int d q \delta(k-q)\left\{g_{k}^{r *} g_{k}^{r^{\prime}} e^{i \omega_{k}\left(t-t^{\prime}\right)} n_{\beta}\left(\omega_{k}\right)\right. \\
\left.+g_{k}^{r} g_{k}^{r^{\prime} *} e^{-i \omega_{k}\left(t-t^{\prime}\right)}\left[n_{\beta}\left(\omega_{k}\right)+1\right]\right\}, \\
C_{\beta}\left(r-r^{\prime}, t-t^{\prime}\right) \\
=\int d k\left|g_{k}\right|^{2}\left\{e^{i\left[\omega_{k}\left(t-t^{\prime}\right)-k\left(r-r^{\prime}\right)\right]} n_{\beta}\left(\omega_{k}\right)\right. \\
\left.\quad+e^{-i\left[\omega_{k}\left(t-t^{\prime}\right)-k\left(r-r^{\prime}\right)\right]}\left[n_{\beta}\left(\omega_{k}\right)+1\right]\right\},
\end{gathered}
$$

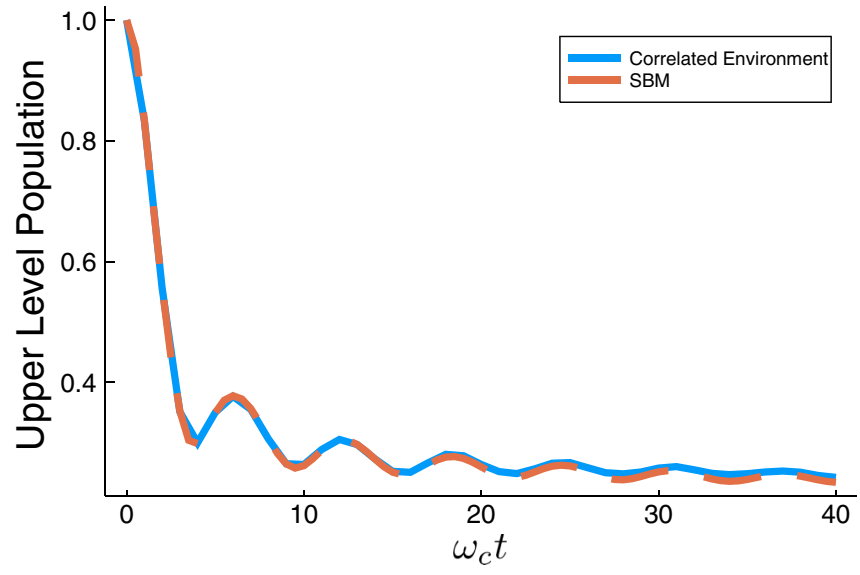

FIG. 16. Dynamics of the up state $\left|\uparrow_{z}\right\rangle$ of a SBM compared with the dynamics of the upper eigenstate of the correlated environment model for the corresponding parameters $\left(k_{c}=1, c=1, J=0.25\right.$, and $\alpha=0.2$ ) with a large separation $R=200$ between the two sites of the system. The two dynamics are the same.

$$
\begin{aligned}
C_{\beta}\left(r-r^{\prime}, t-t^{\prime}\right) \\
=\int d k J\left(\omega_{k}\right)\left[\operatorname{coth}\left(\frac{\beta \omega_{k}}{2}\right) \cos \left[\omega_{k}\left(t-t^{\prime}\right)-k\left(r-r^{\prime}\right)\right]\right. \\
\left.\quad-i \sin \left[\omega_{k}\left(t-t^{\prime}\right)-k\left(r-r^{\prime}\right)\right]\right],
\end{aligned}
$$

with the bath spectral density $J\left(\omega_{k}\right)=\left|g_{k}\right|^{2}$. This bath correlation function has a triple dependence on temperature $\beta$, time $t-t^{\prime}$, and space $r-r^{\prime}$.

\section{APPENDIX B: MAPPING TO SBM FOR $N=2$}

The $N=2$ case is a specific case where, because of the symmetry around the midpoint between the two sites, the problem presented in this paper can be written in the form of a SBM with an effective spectral density that depends explicitly on the site separation. Consider the interaction Hamiltonian $\hat{H}_{\text {int }}$ in Eq. (2) in the case of a two-site system with intersite

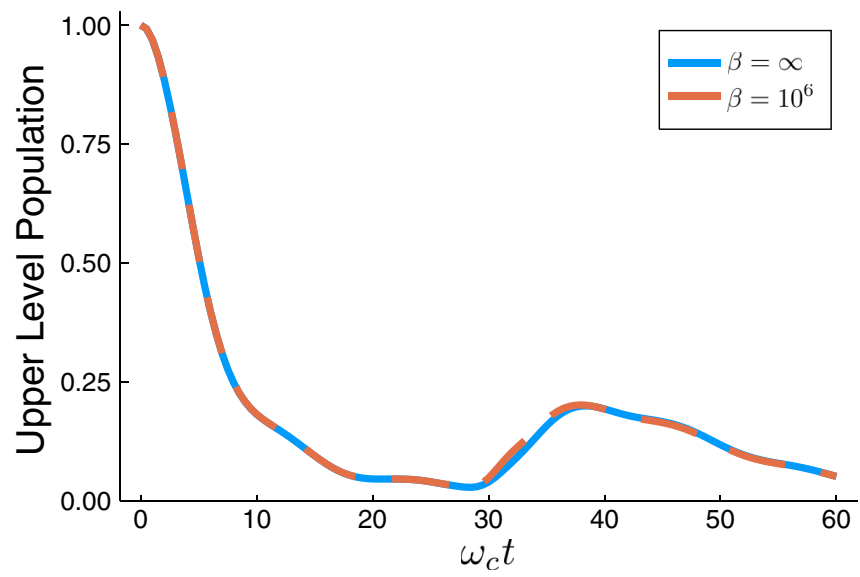

FIG. 17. Upper level population for zero temperature (solid line) and $\beta=10^{6}$ (dashed line). The other parameters are $\alpha=0.03$, $J=0.25, c=1$, and $k_{c}=1$. The dynamics obtained with the zerotemperature and finite-temperature algorithms are identical. 


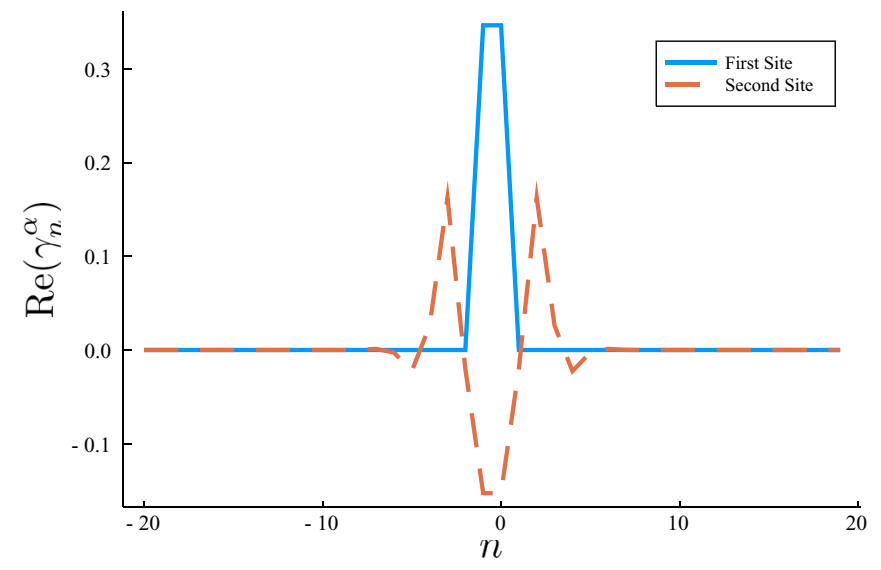

(a)

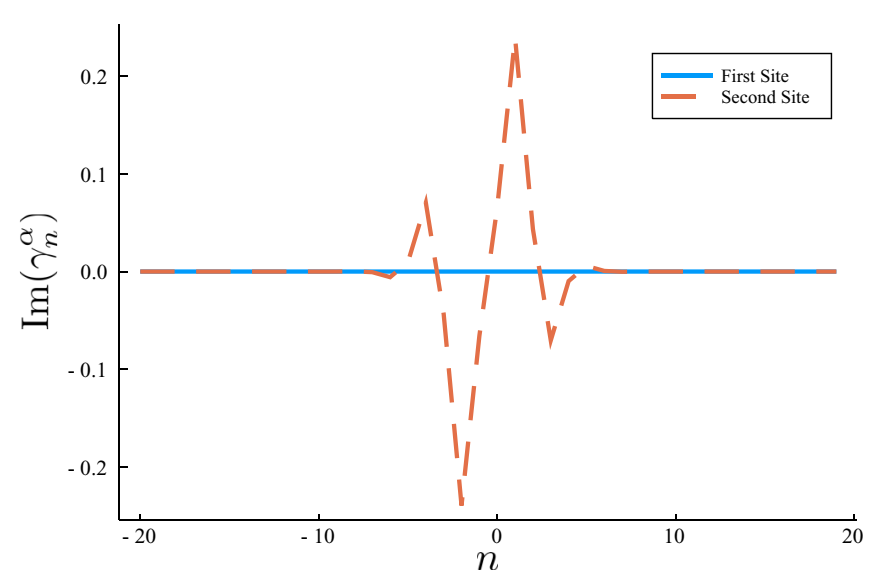

(b)

FIG. 18. (a) Real part of the zero-temperature couplings for the two sites of the system as a function of the chain modes. (b) Imaginary part of the zero-temperature couplings. The negative values along the $x$ axis correspond to the chain of negative wave vectors and the positive values to positive wave vectors. The parameters are $\alpha=0.12, k_{c}=1, c=1, J=0.25$, and $R=5$.

distance $R$, where we have

$$
\begin{aligned}
\hat{H}_{\text {int }} & =\sum_{\alpha}|\alpha\rangle\langle\alpha| \int_{-k_{c}}^{k_{c}}\left(g_{k}^{\alpha} \hat{a}_{k}+g_{k}^{\alpha *} \hat{a}_{k}^{\dagger}\right) d k \\
& =\sum_{\alpha}|\alpha\rangle\langle\alpha| \int_{0}^{k_{c}}\left[g_{k}^{\alpha}\left(\hat{a}_{k}+\hat{a}_{-k}^{\dagger}\right)+g_{k}^{\alpha *}\left(\hat{a}_{k}^{\dagger}+\hat{a}_{-k}\right)\right] d k
\end{aligned}
$$

We can introduce a new set of vibrational modes, the symmetric mode $\hat{c}_{k}$ and the antisymmetric mode $\hat{d}_{k}$,

$$
\begin{aligned}
& \hat{c}_{k}=\frac{\hat{a}_{k}+\hat{a}_{-k}}{\sqrt{2}}, \\
& \hat{d}_{k}=\frac{\hat{a}_{k}-\hat{a}_{-k}}{\sqrt{2}} .
\end{aligned}
$$

Hence, the interaction Hamiltonian becomes

$$
\begin{aligned}
\hat{H}_{\mathrm{int}}= & \sum_{\alpha}|\alpha\rangle\langle\alpha| \int_{0}^{k_{c}}\left[g_{k}^{\alpha} \sqrt{2}\left(\hat{c}_{k}+\hat{c}_{k}^{\dagger}\right)+g_{k}^{\alpha *} \sqrt{2}\left(\hat{c}_{k}+\hat{c}_{k}^{\dagger}\right)\right. \\
& \left.+g_{k}^{\alpha} \sqrt{2}\left(\hat{d}_{k}-\hat{d}_{k}^{\dagger}\right)-g_{k}^{\alpha *} \sqrt{2}\left(\hat{d}_{k}-\hat{d}_{k}^{\dagger}\right)\right] d k
\end{aligned}
$$

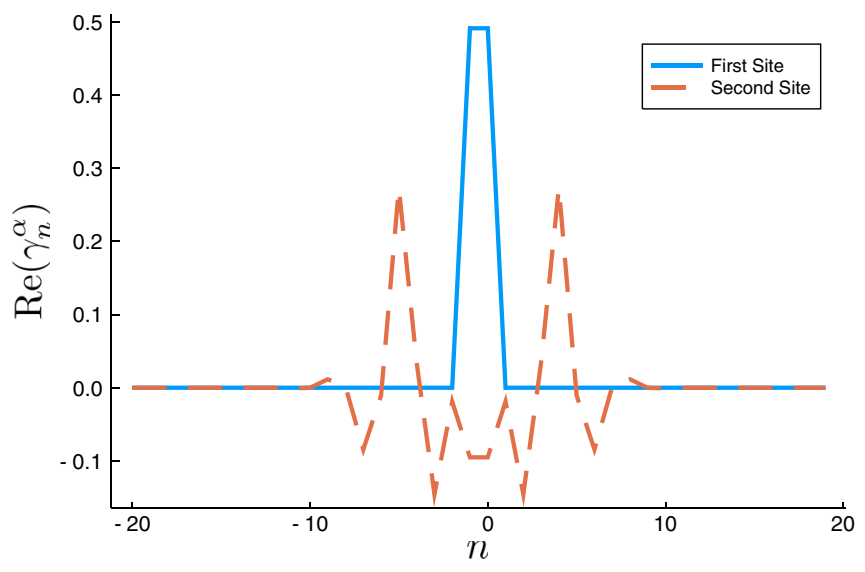

(a)

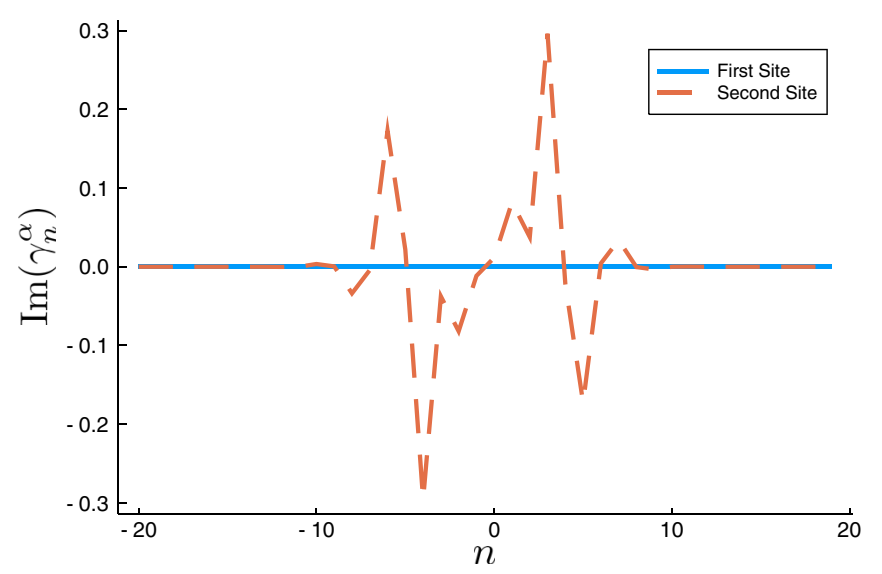

(b)

FIG. 19. (a) Real part of the finite-temperature couplings for the two sites of the system as a function of the chain modes. (b) Imaginary part of the finite-temperature couplings. The negative values along the $x$ axis correspond to the chain of negative wave vectors and the positive values to positive wave vectors. The parameters are the same as in Fig. 11, and in particular $\beta=0.5$.

We choose the origin of position at the midpoint between the two sites so that

$$
\begin{aligned}
& \hat{H}_{\mathrm{int}}=(|-R / 2\rangle\langle-R / 2|+| R / 2\rangle\langle R / 2|) \\
& \times \int_{0}^{k_{c}} 2 \sqrt{2} g_{k} \cos \left(\frac{k R}{2}\right)\left(\hat{c}_{k}+\hat{c}_{k}^{\dagger}\right) d k \\
&+(|-R / 2\rangle\langle-R / 2|-| R / 2\rangle\langle R / 2|) \\
& \times \int_{0}^{k_{c}} 2 \sqrt{2} i g_{k} \sin \left(\frac{k R}{2}\right)\left(\hat{d}_{k}^{\dagger}-\hat{d}_{k}\right) d k \\
& \hat{H}_{\mathrm{int}}=\hat{\mathbb{1}}_{S} \text { const }+\hat{\sigma}_{z} \int_{0}^{k_{c}} 2 \sqrt{2} i g_{k} \sin \left(\frac{k R}{2}\right)\left(\hat{d}_{k}^{\dagger}-\hat{d}_{k}\right) d k
\end{aligned}
$$

Therefore, the system only couples to the antisymmetric vibration modes and thus corresponds to a SBM with an effective spectral density $J_{\text {eff }}(k)=8\left|g_{k}\right|^{2} \sin ^{2}\left(\frac{k R}{2}\right)$. However, for larger values of $N$ it is no longer possible to map the system to a SBM. This is similar to the spin mapping presented in [18]. 


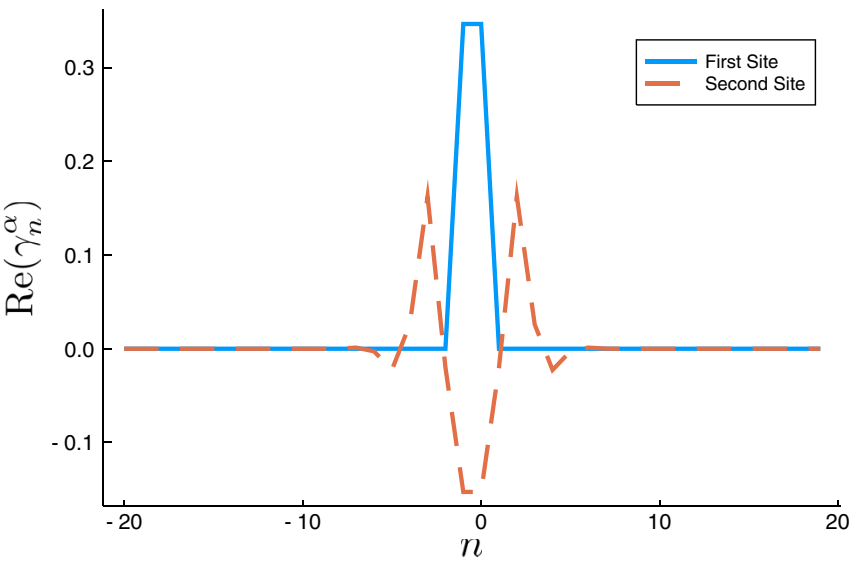

(a)

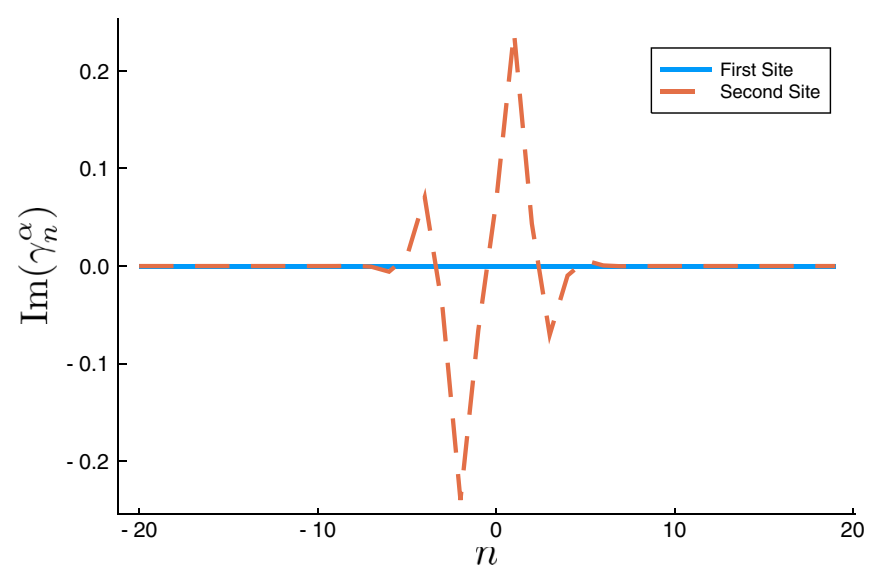

(b)

FIG. 20. (a) Real part of the finite-temperature couplings for the two sites of the system as a function of the chain modes at $\beta=10^{6}$. (b) Imaginary part of the zero-temperature couplings at $\beta=10^{6}$. The behavior is identical to that obtained with the zero-temperature algorithm. The negative values along the $x$ axis correspond to the chain of negative wave vectors and the positive values to positive wave vectors. The other parameters are the same as in Fig. 18.

\section{APPENDIX C: LIMIT CASES}

\section{Large separation}

According to the coupling structure presented in Sec. III A, the farther away the two sites of the system are, the less the second site interacts with the beginning of the chain. Thus, we can expect that for infinite separation when $R \rightarrow \infty$ this system will behave like a SBM. Figure 16 shows the comparison between the SBM and the infinite-separation case.

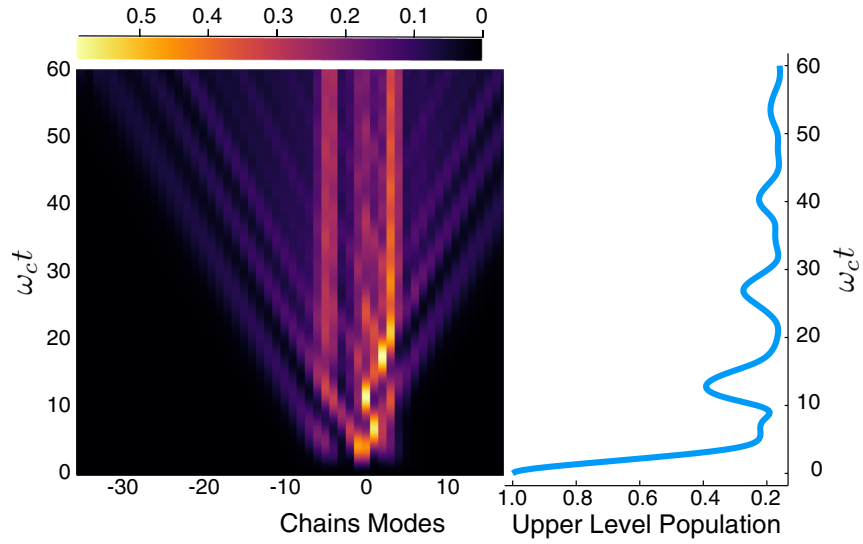

(a)

(b)

FIG. 21. (a) Heatmap showing the propagation of bath excitations along the chains. (b) System eigenstates population for an initial state in the upper eigenstate. The separation between the two sites is $R=20$, their coupling is $J=0.25$, the speed of sound is $c=2$, $\alpha=0.12$, and $k_{c}=1$. We can definitely see a revival of population at a time consistent with the amount of time needed for a bosonic excitation to travel into the bath from one system's site to the other.

\section{Low temperature}

The finite-temperature effective spectral density $J_{\beta}(k)$ converges toward the zero-temperature one when $\beta \rightarrow \infty$ as its value for a negative wave vector becomes uniformly null. Hence, the quantities calculated using this finite-temperature function should all converge toward their zero-temperature counterparts when $\beta$ is increased. Figure 17 shows that the population dynamics of the zero-temperature case is recovered.

For the same reasons the couplings $\gamma_{n}(R)$ determined with the finite-temperature spectral density should become identical to the zero-temperature ones calculated with the spectral density $J(k)$. This was already shown in Sec. IV B with the absolute values of the coupling constants $\gamma_{n}(R)$ as shown in Fig. 12. The real and imaginary parts of the zero-temperature coupling constants for the two sites are presented in Figs. 18 and 19. Figure 19 shows the coupling constants at a finite temperature $\beta=0.5$ for comparison. The finite-temperature couplings for a large $\beta$ are presented in Fig. 20 and show that the finite-temperature coupling coefficients converge to the zero-temperature ones when the limit $\beta \rightarrow \infty$ is taken.

\section{APPENDIX D: BATH DYNAMICS}

The unannotated version of the bath dynamics displayed in Fig. 7, presenting several consecutive revivals of the upper eigenstate population for $R=20$ and $c=2$, is shown in Fig. 21.
[1] H.-P. Breuer and F. Petruccione, The Theory of Open Quantum Systems (Oxford University Press, Oxford, 2007).

[2] U. Weiss, Quantum Dissipative Systems, 4th ed. (World Scientific, Singapore, 2012).
[3] K. Blum, Density Matrix Theory and Applications, 3rd ed., Springer Series on Atomic, Optical, and Plasma Physics Vol. 64 (Springer, Berlin, 2012).

[4] P. Strasberg, G. Schaller, N. Lambert, and T. Brandes, Nonequilibrium thermodynamics in the strong coupling and 
non-Markovian regime based on a reaction coordinate mapping, New J. Phys. 18, 073007 (2016).

[5] G. L. Giorgi and S. Campbell, Correlation approach to work extraction from finite quantum systems, J. Phys. B 48, 035501 (2015).

[6] S. Oviedo-Casado, J. Prior, A. W. Chin, R. Rosenbach, S. F. Huelga, and M. B. Plenio, Phase-dependent exciton transport and energy harvesting from thermal environments, Phys. Rev. A 93, 020102(R) (2016).

[7] S. Vinjanampathy and J. Anders, Quantum thermodynamics, Contemp. Phys. 57, 545 (2016).

[8] R. Kosloff, Quantum thermodynamics: A dynamical viewpoint, Entropy 15, 2100 (2013).

[9] R. E. Blackenship, Molecular Mechanisms of Photosynthesis (Wiley, New York, 2002).

[10] G. S. Engel, T. R. Calhoun, E. L. Read, T.-K. Ahn, T. Mančal, Y.-C. Cheng, R. E. Blankenship, and G. R. Fleming, Evidence for wavelike energy transfer through quantum coherence in photosynthetic systems, Nature (London) 446, 782 (2007).

[11] E. Collini, C. Y. Wong, K. E. Wilk, P. M. G. Curmi, P. Brumer, and G. D. Scholes, Coherently wired light-harvesting in photosynthetic marine algae at ambient temperature, Nature (London) 463, 644 (2010).

[12] A. Chin, J. Prior, R. Rosenbach, F. Caycedo-Soler, S. F. Huelga, and M. B. Plenio, The role of non-equilibrium vibrational structures in electronic coherence and recoherence in pigmentprotein complexes, Nat. Phys. 9, 113 (2013).

[13] C. Kreisbeck and T. Kramer, Long-lived electronic coherence in dissipative exciton dynamics of light-harvesting complexes, J. Phys. Chem. Lett. 3, 2828 (2012).

[14] F. Müh and A. Zouni, The nonheme iron in photosystem II, Photosynth. Res. 116, 295 (2013).

[15] O. Bozovic, C. Zanobini, A. Gulzar, B. Jankovic, D. Buhrke, M. Post, S. Wolf, G. Stock, and P. Hamm, Real-time observation of ligand-induced allosteric transitions in a PDZ domain, Proc. Natl. Acad. Sci. USA 117, 26031 (2020).

[16] J. Guo and H.-X. Zhou, Protein allostery and conformational dynamics, Chem. Rev. 116, 6503 (2016).

[17] M. R. Jørgensen and F. A. Pollock, A discrete memory-kernel for multitime correlations in non-Markovian quantum processes, Phys. Rev. A 102, 052206 (2020).

[18] A. Strathearn, P. Kirton, D. Kilda, J. Keeling, and B. W. Lovett, Efficient non-Markovian quantum dynamics using timeevolving matrix product operators, Nat. Commun. 9, 3322 (2018).

[19] D. Gribben, A. Strathearn, J. Iles-Smith, D. Kilda, A. Nazir, B. W. Lovett, and P. Kirton, Exact quantum dynamics in structured environments, Phys. Rev. Research 2, 013265 (2020).

[20] M. R. Jørgensen and F. A. Pollock, Exploiting the Causal Tensor Network Structure of Quantum Processes to Efficiently Simulate Non-Markovian Path Integrals, Phys. Rev. Lett. 123, 240602 (2019).

[21] G. E. Fux, E. P. Butler, P. R. Eastham, B. W. Lovett, and J. Keeling, Efficient Exploration of Hamiltonian Parameter Space for Optimal Control of Non-Markovian Open Quantum Systems, Phys. Rev. Lett. 126, 200401 (2021).

[22] A. W. Chin, A. Rivas, S. F. Huelga, and M. B. Plenio, Exact mapping between system-reservoir quantum models and semiinfinite discrete chains using orthogonal polynomials, J. Math. Phys. 51, 092109 (2010).
[23] H.-D. Meyer, Studying molecular quantum dynamics with the multiconfiguration time-dependent Hartree method, WIRES: Comput. Mol. Sci. 2, 351 (2012).

[24] A. J. Dunnett and A. W. Chin, Simulating quantum vibronic dynamics at finite temperatures with many body wave functions at $0 \mathrm{~K}$, Front. Chem. 8, 600731 (2021).

[25] G. Vidal, Efficient Simulation of One-Dimensional Quantum Many-Body Systems, Phys. Rev. Lett. 93, 040502 (2004).

[26] Y.-Y. Shi, L.-M. Duan, and G. Vidal, Classical simulation of quantum many-body systems with a tree tensor network, Phys. Rev. A 74, 022320 (2006).

[27] J. Haegeman, C. Lubich, I. Oseledets, B. Vandereycken, and F. Verstraete, Unifying time evolution and optimization with matrix product states, Phys. Rev. B 94, 165116 (2016).

[28] J. Prior, A. W. Chin, S. F. Huelga, and M. B. Plenio, Efficient Simulation of Strong System-Environment Interactions, Phys. Rev. Lett. 105, 050404 (2010).

[29] M. P. Woods, M. Cramer, and M. B. Plenio, Simulating Bosonic Baths with Error Bars, Phys. Rev. Lett. 115, 130401 (2015).

[30] M. Mohseni, Y. Omar, G. S. Engel, and M. B. Plenio, Quantum Effects in Biology (Cambridge University Press, Cambridge, 2014).

[31] D. Tamascelli, A. Smirne, J. Lim, S. F. Huelga, and M. B. Plenio, Efficient Simulation of Finite-Temperature Open Quantum Systems, Phys. Rev. Lett. 123, 090402 (2019).

[32] S. Paeckel, T. Köhler, A. Swoboda, S. R. Manmana, U. Schollwöck, and C. Hubig, Time-evolution methods for matrixproduct states, Ann. Phys. (NY) 411, 167998 (2019).

[33] H. Ollivier, D. Poulin, and W. H. Zurek, Environment as a witness: Selective proliferation of information and emergence of objectivity in a quantum universe, Phys. Rev. A 72, 042113 (2005).

[34] D. Tamascelli, Excitation dynamics in chain-mapped environments, Entropy 22, 1320 (2020).

[35] C. Olbrich, J. Strümpfer, K. Schulten, and U. Kleinekathöfer, Quest for spatially correlated fluctuations in the FMO light-harvesting complex, J. Phys. Chem. B 115, 758 (2011).

[36] T. J. Zuehlsdorff, S. V. Shedge, S.-Y. Lu, H. Hong, V. P. Aguirre, L. Shi, and C. M. Isborn, Vibronic and environmental effects in simulations of optical spectroscopy, Annu. Rev. Phys. Chem. 72, 165 (2021).

[37] T. Renger, A. Klinger, F. Steinecker, M. Schmidt am Busch, J. Numata, and F. Müh, Normal mode analysis of the spectral density of the Fenna-Matthews-Olson light-harvesting protein: How the protein dissipates the excess energy of excitons, J. Phys. Chem. B 116, 14565 (2012).

[38] S. E. Morgan, D. J. Cole, and A. W. Chin, Nonlinear network model analysis of vibrational energy transfer and localisation in the Fenna-Matthews-Olson complex, Sci. Rep. 6, 36703 (2016).

[39] M. L. Chaillet, F. Lengauer, J. Adolphs, F. Müh, A. S. Fokas, D. J. Cole, A. W. Chin, and T. Renger, Static disorder in excitation energies of the Fenna-Matthews-Olson protein: Structure-based theory meets experiment, J. Phys. Chem. Lett. 11, 10306 (2020).

[40] A. S. Fokas, D. J. Cole, N. D. Hine, S. A. Wells, M. C. Payne, and A. W. Chin, Evidence of correlated static disorder in the Fenna-Matthews-Olson complex, J. Phys. Chem. Lett. 8, 2350 (2017). 